\title{
A Comprehensive Study on Gamma-Ray Exposure Build-Up Factors and Fast Neutron Removal Cross Sections of Fly-Ash Bricks
}

\author{
Vishwanath P. Singh ${ }^{1,2}$ and N. M. Badiger ${ }^{1}$ \\ ${ }^{1}$ Department of Physics, Karnatak University, Dharwad 580003, India \\ ${ }^{2}$ Health Physics Section, Kaiga Atomic Power Station-3\&4, NPCIL, Karwar 581400, India \\ Correspondence should be addressed to Vishwanath P. Singh; kudphyvps@rediffmail.com
}

Received 31 March 2013; Accepted 17 June 2013

Academic Editor: Shaomin Liu

Copyright (C) 2013 V. P. Singh and N. M. Badiger. This is an open access article distributed under the Creative Commons Attribution License, which permits unrestricted use, distribution, and reproduction in any medium, provided the original work is properly cited.

\begin{abstract}
Geometric progression (GP) method was utilized to investigate gamma-ray exposure build-up factors of fly-ash bricks for energies from 0.015 to $15 \mathrm{MeV}$ up to $40 \mathrm{mfp}$ penetration depth. The EBFs of the fly-ash bricks are dependent upon the photon energy, penetration depths, and the chemical compositions of the elements. Appreciable variations in exposure build-up factor (EBF) are noted for the fly-ash bricks. The EBFs were found to be small in low and high photon energy regions whereas very large in medium energy region. EBF of the bricks is inversely proportional to equivalent atomic number below $10 \mathrm{mfp}$ for entire energy region of interest 0.015 to $15 \mathrm{MeV}$. The EBFs of fly-ash, brick of mud, and common brick were similar at $1.5 \mathrm{MeV}$ photon energy. The EBF of the fly-ash bricks was found to be higher than that of the brick of mud, and common brick. The fast neutron removal cross sections of the fly-ash bricks, brick of mud, and common bricks were also calculated which were found to be in the same order. It is expected that this study should be very directly useful for shielding effectiveness of fly-ash brick materials and dose estimation.
\end{abstract}

\section{Introduction}

Safety inside residential and nonresidential building against the radiation is evaluated by the shielding properties by the parameters such as mass attenuation coefficients, energy absorption coefficients, and half-value layer. Gamma-ray interacts with material by photoelectric absorption, compton scattering, and pair production, which depends upon photon energy and element compositions. The intensity of a gammaray beam through a medium follows the Lambert Beer law under three conditions namely, (i) monochromatic rays, (ii) thin absorbing material, and (iii) narrow beam geometry. In case, any of the above conditions is not being met, this law is no longer applicable. The law can be applicable by using a correction factor, called as "build-up factor."

The concept of build-up factor was introduced in late 1950 [2] by obtaining experimentally the build-up factor at $1.25 \mathrm{MeV}$ gamma-ray energy for water up to $16 \mathrm{mfp}$. The importance of build-up factor in attenuation studies was further recognized for multienergy gamma-rays with poor geometry [3]. Since 1950, due to the availability of reasonably accurate values of attenuation coefficients and cross section of the various mediums, a great progress has been made in the computation of build-up factor in different types of materials such as medical, dosimetric, shielding, and radiation protection.

The build-up factor is a dimensionless multiplication factor which corrects the response of uncollided photon beam. The build-up is defined as the ratio of total value of specified radiation quantity at any point to the contribution to that value from radiation reaching the point without having undergone a collision. There are two types of buildup factors which are the quantity of interest: (a) the absorbed or deposited energy in the interacting materials and detector response function is that of absorption in the interacting medium; (b) the exposure build-up factor in which quality of interest is the exposure and detector response function is that of absorption in air [4]. The build-up factors have been computed by various codes such as PALLAS [5], ADJMONI $[6,7]$, ASFIT [8], and EGS4 [9]. These codes are using 
TABLE 1: Elemental compositions of fly-ash bricks, brick of mud, and common brick [1].

\begin{tabular}{|c|c|c|c|c|c|c|c|c|c|c|}
\hline \multirow{3}{*}{$\begin{array}{l}\text { Element } \\
\text { Density }\left(\mathrm{g} \mathrm{cm}^{-3}\right)\end{array}$} & \multicolumn{10}{|c|}{ Elemental composition (\% by weight) } \\
\hline & FAB1 & FAB2 & FAB3 & FAB4 & FAB5 & FAB6 & FAB7 & FAB8 & $\mathrm{BOM}$ & $\mathrm{COM}$ \\
\hline & 1.87 & 1.81 & 1.76 & 1.72 & 1.85 & 1.79 & 1.74 & 1.70 & 1.82 & 1.87 \\
\hline $\mathrm{H}$ & 0.09 & 0.09 & 0.09 & 0.09 & 0.09 & 0.09 & 0.09 & 0.09 & - & 0.24 \\
\hline $\mathrm{C}$ & 3.69 & 3.41 & 3.13 & 2.84 & 1.70 & 1.70 & 1.70 & 1.70 & - & 0.06 \\
\hline $\mathrm{N}$ & - & - & - & - & - & - & - & - & - & 0.01 \\
\hline $\mathrm{O}$ & 48.71 & 48.70 & 48.69 & 48.68 & 49.96 & 49.77 & 49.58 & 49.39 & 54.6 & 45.35 \\
\hline $\mathrm{Na}$ & 0.01 & 0.01 & 0.01 & 0.01 & 0.11 & 0.09 & 0.08 & 0.07 & 0.11 & 0.01 \\
\hline $\mathrm{Mg}$ & 1.07 & 1.03 & 0.99 & 0.94 & 0.77 & 0.77 & 0.77 & 0.77 & 0.11 & 0.04 \\
\hline $\mathrm{Al}$ & 11.77 & 11.13 & 10.5 & 9.86 & 10.78 & 10.28 & 9.79 & 9.29 & 4.95 & 7.88 \\
\hline $\mathrm{Si}$ & 23.22 & 23.09 & 22.96 & 22.83 & 25.08 & 24.68 & 24.29 & 23.9 & 5.96 & 20.78 \\
\hline $\mathrm{P}$ & - & - & - & - & 0.07 & 0.06 & 0.05 & 0.04 & - & - \\
\hline S & 0.04 & 0.04 & 0.03 & 0.03 & 0.02 & 0.02 & 0.02 & 0.02 & - & 0.02 \\
\hline $\mathrm{K}$ & 0.42 & 0.80 & 1.18 & 1.56 & 0.44 & 0.82 & 1.20 & 1.57 & - & 2.66 \\
\hline $\mathrm{Ca}$ & 6.92 & 6.77 & 6.61 & 6.45 & 7.32 & 7.10 & 6.88 & 6.67 & 34.01 & 4.75 \\
\hline $\mathrm{Ti}$ & 0.01 & 0.01 & 0.01 & 0.01 & 0.57 & 0.49 & 0.41 & 0.33 & - & 0.09 \\
\hline $\mathrm{Mn}$ & 0.01 & 0.01 & 0.01 & 0.01 & 0.01 & 0.01 & 0.01 & 0.01 & - & 0.17 \\
\hline $\mathrm{Fe}$ & 4.07 & 4.95 & 5.84 & 6.73 & 3.12 & 4.15 & 5.17 & 6.19 & 0.30 & 17.96 \\
\hline
\end{tabular}

TABLE 2: Equivalent atomic number of fly-ash bricks and other bricks.

\begin{tabular}{|c|c|c|c|c|c|c|c|c|c|c|}
\hline \multirow{2}{*}{ Energy $(\mathrm{MeV})$} & \multicolumn{10}{|c|}{ Equivalent atomic number $\left(Z_{\mathrm{eq}}\right)$} \\
\hline & FAB1 & FAB2 & FAB3 & FAB4 & FAB5 & FAB6 & FAB7 & FAB8 & $\mathrm{BOM}$ & $\mathrm{COB}$ \\
\hline 0.015 & 13.16 & 13.39 & 13.61 & 13.81 & 13.12 & 13.35 & 13.58 & 13.79 & 14.61 & 15.97 \\
\hline 0.02 & 13.32 & 13.56 & 13.78 & 14.00 & 13.27 & 13.51 & 13.75 & 13.97 & 14.78 & 16.22 \\
\hline 0.03 & 13.50 & 13.74 & 13.98 & 14.20 & 13.43 & 13.69 & 13.93 & 14.16 & 14.95 & 16.50 \\
\hline 0.04 & 13.61 & 13.87 & 14.11 & 14.34 & 13.53 & 13.80 & 14.06 & 14.30 & 15.06 & 16.67 \\
\hline 0.05 & 13.69 & 13.95 & 14.20 & 14.44 & 13.62 & 13.89 & 14.15 & 14.40 & 15.14 & 16.79 \\
\hline 0.06 & 13.76 & 14.03 & 14.27 & 14.51 & 13.69 & 13.96 & 14.21 & 14.47 & 15.20 & 16.88 \\
\hline 0.08 & 13.85 & 14.13 & 14.37 & 14.61 & 13.77 & 14.04 & 14.31 & 14.56 & 15.28 & 17.02 \\
\hline 0.1 & 13.91 & 14.21 & 14.43 & 14.68 & 13.83 & 14.10 & 14.37 & 14.63 & 15.33 & 17.12 \\
\hline 0.15 & 14.02 & 14.33 & 14.54 & 14.80 & 13.91 & 14.19 & 14.47 & 14.74 & 15.41 & 17.24 \\
\hline 0.2 & 14.07 & 14.33 & 14.60 & 14.87 & 13.96 & 14.25 & 14.53 & 14.81 & 15.46 & 17.31 \\
\hline 0.3 & 14.13 & 14.40 & 14.67 & 14.95 & 14.02 & 14.31 & 14.60 & 14.89 & 15.51 & 17.40 \\
\hline 0.4 & 14.16 & 14.44 & 14.72 & 15.00 & 14.05 & 14.34 & 14.64 & 14.93 & 15.54 & 17.45 \\
\hline 0.5 & 14.18 & 14.46 & 14.74 & 15.02 & 14.07 & 14.36 & 14.66 & 14.96 & 15.55 & 17.48 \\
\hline 0.6 & 14.19 & 14.47 & 14.76 & 15.04 & 14.08 & 14.38 & 14.67 & 14.97 & 15.56 & 17.50 \\
\hline 0.8 & 14.20 & 14.48 & 14.77 & 15.05 & 14.08 & 14.39 & 14.68 & 14.98 & 15.57 & 17.52 \\
\hline 1 & 14.20 & 14.49 & 14.77 & 15.05 & 14.09 & 14.39 & 14.69 & 14.99 & 15.57 & 17.52 \\
\hline 1.5 & 12.22 & 12.44 & 12.65 & 12.86 & 12.23 & 12.44 & 12.66 & 12.87 & 13.66 & 15.15 \\
\hline 2 & 11.69 & 11.85 & 12.01 & 12.16 & 11.74 & 11.89 & 12.04 & 12.19 & 13.00 & 14.01 \\
\hline 3 & 11.56 & 11.71 & 11.85 & 11.99 & 11.62 & 11.75 & 11.89 & 12.02 & 12.82 & 13.70 \\
\hline 4 & 11.52 & 11.66 & 11.80 & 11.94 & 11.57 & 11.71 & 11.84 & 11.97 & 12.76 & 13.63 \\
\hline 5 & 11.51 & 11.64 & 11.78 & 11.92 & 11.56 & 11.70 & 11.83 & 11.96 & 12.75 & 13.59 \\
\hline 6 & 11.50 & 11.63 & 11.77 & 11.91 & 11.56 & 11.69 & 11.81 & 11.94 & 12.73 & 13.57 \\
\hline 8 & 11.49 & 11.62 & 11.76 & 11.90 & 11.54 & 11.67 & 11.80 & 11.93 & 12.72 & 13.54 \\
\hline 10 & 11.48 & 11.61 & 11.75 & 11.89 & 11.54 & 11.67 & 11.79 & 11.92 & 12.71 & 13.53 \\
\hline 15 & 11.47 & 11.61 & 11.74 & 11.88 & 11.53 & 11.66 & 11.79 & 11.91 & 12.70 & 13.51 \\
\hline
\end{tabular}

an accurate algorithmic for the Klein-Nishina cross section which eliminated other sources of errors.

The compilation for build-up factors by various codes was reported in ANSI/ANS-6.4.3-1991 by American Nuclear Society [10]. The data in the report covers energy range from 0.015 to $15 \mathrm{MeV}$ up to penetration depth of 40 mean free path (mfp). The build-up factors in the ANS-6.4.3 are for 23 elements of atomic number, $Z=4$ to 92 . The build-up factors of ANS-6.4.3 can be calculated by invariant embedding [11, 12]. Harima et al. [13] developed a fitting formula, called geometric progression (GP), which gave build-up factors of the good agreement with the ANS-6.4.3. The GP fitting is more accurate than three exponential fit in the water medium. The GP fitting formula is known to be accurate within the 
TABLE 3: GP fitting parameters for FAB1 and FAB2.

\begin{tabular}{|c|c|c|c|c|c|}
\hline Energy $(\mathrm{MeV})$ & $b$ & $c$ & $a$ & $X_{K}$ & $d$ \\
\hline & \multicolumn{5}{|c|}{ FAB1 } \\
\hline 0.015 & 1.028 & 0.386 & 0.215 & 14.919 & -0.146 \\
\hline 0.02 & 1.062 & 0.398 & 0.206 & 15.078 & -0.116 \\
\hline 0.03 & 1.201 & 0.408 & 0.207 & 14.534 & -0.110 \\
\hline 0.04 & 1.421 & 0.476 & 0.180 & 14.500 & -0.100 \\
\hline 0.05 & 1.677 & 0.605 & 0.124 & 15.483 & -0.065 \\
\hline 0.06 & 1.963 & 0.701 & 0.095 & 14.727 & -0.053 \\
\hline 0.08 & 2.435 & 0.851 & 0.055 & 14.413 & -0.048 \\
\hline 0.1 & 2.646 & 1.026 & 0.014 & 13.593 & -0.029 \\
\hline 0.15 & 2.731 & 1.272 & -0.042 & 10.413 & -0.006 \\
\hline 0.2 & 2.670 & 1.371 & -0.057 & 7.813 & -0.005 \\
\hline 0.3 & 2.476 & 1.464 & -0.077 & 17.191 & 0.013 \\
\hline 0.4 & 2.350 & 1.467 & -0.080 & 16.046 & 0.016 \\
\hline 0.5 & 2.247 & 1.451 & -0.079 & 16.302 & 0.018 \\
\hline 0.6 & 2.171 & 1.422 & -0.076 & 18.225 & 0.021 \\
\hline 0.8 & 2.049 & 1.383 & -0.073 & 15.355 & 0.021 \\
\hline 1 & 1.976 & 1.323 & -0.063 & 16.064 & 0.019 \\
\hline 1.5 & 1.864 & 1.232 & -0.048 & 14.866 & 0.017 \\
\hline 2 & 1.789 & 1.155 & -0.033 & 15.608 & 0.011 \\
\hline 3 & 1.678 & 1.061 & -0.012 & 13.922 & -0.001 \\
\hline 4 & 1.602 & 0.995 & 0.005 & 12.945 & -0.009 \\
\hline 5 & 1.537 & 0.948 & 0.020 & 12.601 & -0.021 \\
\hline 6 & 1.474 & 0.924 & 0.027 & 11.700 & -0.022 \\
\hline 8 & 1.406 & 0.902 & 0.033 & 13.689 & -0.027 \\
\hline 10 & 1.345 & 0.881 & 0.042 & 13.141 & -0.033 \\
\hline \multirow[t]{2}{*}{15} & 1.258 & 0.835 & 0.061 & 14.297 & -0.052 \\
\hline & \multicolumn{5}{|c|}{ FAB2 } \\
\hline 0.015 & 1.027 & 0.376 & 0.228 & 14.103 & -0.155 \\
\hline 0.02 & 1.058 & 0.408 & 0.197 & 15.983 & -0.113 \\
\hline 0.03 & 1.190 & 0.402 & 0.212 & 14.267 & -0.113 \\
\hline 0.04 & 1.397 & 0.466 & 0.185 & 14.389 & -0.104 \\
\hline 0.05 & 1.646 & 0.583 & 0.133 & 15.174 & -0.071 \\
\hline 0.06 & 1.890 & 0.700 & 0.093 & 15.276 & -0.052 \\
\hline 0.08 & 2.374 & 0.827 & 0.061 & 14.429 & -0.051 \\
\hline 0.1 & 2.586 & 1.001 & 0.016 & 13.739 & -0.032 \\
\hline 0.15 & 2.692 & 1.248 & -0.037 & 10.823 & -0.009 \\
\hline 0.2 & 2.647 & 1.356 & -0.055 & 8.168 & -0.006 \\
\hline 0.3 & 2.462 & 1.454 & -0.076 & 17.822 & 0.013 \\
\hline 0.4 & 2.342 & 1.458 & -0.078 & 16.424 & 0.015 \\
\hline 0.5 & 2.239 & 1.445 & -0.078 & 16.305 & 0.017 \\
\hline 0.6 & 2.162 & 1.420 & -0.076 & 17.592 & 0.020 \\
\hline 0.8 & 2.044 & 1.380 & -0.072 & 15.458 & 0.021 \\
\hline 1 & 1.970 & 1.323 & -0.063 & 16.408 & 0.019 \\
\hline 1.5 & 1.862 & 1.232 & -0.048 & 15.186 & 0.017 \\
\hline 2 & 1.788 & 1.155 & -0.033 & 15.865 & 0.011 \\
\hline 3 & 1.677 & 1.062 & -0.012 & 14.771 & -0.001 \\
\hline 4 & 1.601 & 0.995 & 0.005 & 12.967 & -0.009 \\
\hline 5 & 1.537 & 0.946 & 0.021 & 11.908 & -0.021 \\
\hline 6 & 1.477 & 0.927 & 0.026 & 11.786 & -0.021 \\
\hline 8 & 1.405 & 0.902 & 0.034 & 13.732 & -0.028 \\
\hline 10 & 1.344 & 0.882 & 0.042 & 13.128 & -0.033 \\
\hline 15 & 1.257 & 0.838 & 0.060 & 14.282 & -0.052 \\
\hline
\end{tabular}

estimated uncertainty $(<5 \%)$ and Harima [4] had extensive historical review and reported the current gamma photon
TABLE 4: GP fitting parameters for FAB3 and FAB4.

\begin{tabular}{|c|c|c|c|c|c|}
\hline Energy $(\mathrm{MeV})$ & $b$ & $c$ & $a$ & $X_{K}$ & $d$ \\
\hline & \multicolumn{5}{|c|}{ FAB3 } \\
\hline 0.015 & 1.025 & 0.366 & 0.240 & 13.337 & -0.164 \\
\hline 0.02 & 1.054 & 0.417 & 0.188 & 16.831 & -0.110 \\
\hline 0.03 & 1.180 & 0.396 & 0.216 & 14.016 & -0.116 \\
\hline 0.04 & 1.377 & 0.458 & 0.189 & 14.341 & -0.108 \\
\hline 0.05 & 1.618 & 0.567 & 0.141 & 15.031 & -0.086 \\
\hline 0.06 & 1.854 & 0.683 & 0.099 & 15.149 & -0.055 \\
\hline 0.08 & 2.327 & 0.808 & 0.068 & 14.016 & -0.051 \\
\hline 0.1 & 2.542 & 0.984 & 0.020 & 13.835 & -0.034 \\
\hline 0.15 & 2.667 & 1.232 & -0.034 & 11.093 & -0.010 \\
\hline 0.2 & 2.624 & 1.341 & -0.052 & 8.518 & -0.007 \\
\hline 0.3 & 2.448 & 1.444 & -0.074 & 18.446 & 0.013 \\
\hline 0.4 & 2.333 & 1.449 & -0.077 & 16.798 & 0.015 \\
\hline 0.5 & 2.231 & 1.439 & -0.077 & 16.307 & 0.016 \\
\hline 0.6 & 2.154 & 1.418 & -0.075 & 16.966 & 0.019 \\
\hline 0.8 & 2.039 & 1.377 & -0.071 & 15.559 & 0.021 \\
\hline 1 & 1.964 & 1.323 & -0.063 & 16.748 & 0.020 \\
\hline 1.5 & 1.859 & 1.231 & -0.048 & 15.503 & 0.017 \\
\hline 2 & 1.787 & 1.155 & -0.033 & 16.105 & 0.011 \\
\hline 3 & 1.675 & 1.063 & -0.013 & 15.611 & -0.002 \\
\hline 4 & 1.600 & 0.996 & 0.005 & 12.989 & -0.009 \\
\hline 5 & 1.538 & 0.944 & 0.022 & 11.222 & -0.021 \\
\hline 6 & 1.480 & 0.930 & 0.026 & 11.870 & -0.020 \\
\hline 8 & 1.405 & 0.902 & 0.034 & 13.775 & -0.028 \\
\hline 10 & 1.343 & 0.884 & 0.041 & 13.114 & -0.033 \\
\hline \multirow[t]{2}{*}{15} & 1.255 & 0.841 & 0.059 & 14.267 & -0.052 \\
\hline & \multicolumn{5}{|c|}{ FAB4 } \\
\hline 0.015 & 1.024 & 0.357 & 0.252 & 12.616 & -0.172 \\
\hline 0.02 & 1.051 & 0.426 & 0.179 & 17.628 & -0.108 \\
\hline 0.03 & 1.171 & 0.395 & 0.215 & 14.076 & -0.033 \\
\hline 0.04 & 1.360 & 0.453 & 0.190 & 14.365 & -0.107 \\
\hline 0.05 & 1.592 & 0.553 & 0.147 & 14.928 & -0.080 \\
\hline 0.06 & 1.820 & 0.666 & 0.105 & 15.025 & -0.058 \\
\hline 0.08 & 2.278 & 0.788 & 0.074 & 13.592 & -0.051 \\
\hline 0.1 & 2.494 & 0.964 & 0.025 & 13.941 & -0.037 \\
\hline 0.15 & 2.635 & 1.213 & -0.030 & 11.426 & -0.012 \\
\hline 0.2 & 2.601 & 1.326 & -0.049 & 8.863 & -0.008 \\
\hline 0.3 & 2.434 & 1.435 & -0.072 & 19.060 & 0.013 \\
\hline 0.4 & 2.325 & 1.440 & -0.075 & 17.165 & 0.014 \\
\hline 0.5 & 2.223 & 1.433 & -0.076 & 16.309 & 0.016 \\
\hline 0.6 & 2.146 & 1.415 & -0.075 & 16.510 & 0.018 \\
\hline 0.8 & 2.035 & 1.374 & -0.071 & 15.691 & 0.020 \\
\hline 1 & 1.959 & 1.322 & -0.063 & 16.955 & 0.020 \\
\hline 1.5 & 1.857 & 1.230 & -0.048 & 15.815 & 0.017 \\
\hline 2 & 1.786 & 1.155 & -0.033 & 15.976 & 0.011 \\
\hline 3 & 1.674 & 1.064 & -0.013 & 16.447 & -0.002 \\
\hline 4 & 1.598 & 0.997 & 0.005 & 13.010 & -0.009 \\
\hline 5 & 1.538 & 0.942 & 0.023 & 10.541 & -0.022 \\
\hline 6 & 1.482 & 0.932 & 0.025 & 11.955 & -0.020 \\
\hline 8 & 1.405 & 0.902 & 0.034 & 13.818 & -0.028 \\
\hline 10 & 1.343 & 0.885 & 0.041 & 13.101 & -0.033 \\
\hline 15 & 1.254 & 0.844 & 0.058 & 14.253 & -0.051 \\
\hline
\end{tabular}

build-up factors and applications [14]. Various researchers have investigated gamma-ray build-up factors in different 
TABLE 5: GP fitting parameters for FAB5 and FAB6.

\begin{tabular}{|c|c|c|c|c|c|}
\hline Energy $(\mathrm{MeV})$ & $b$ & $c$ & $a$ & $X_{K}$ & $d$ \\
\hline & \multicolumn{5}{|c|}{ FAB5 } \\
\hline 0.015 & 1.028 & 0.389 & 0.213 & 15.089 & -0.144 \\
\hline 0.02 & 1.062 & 0.395 & 0.209 & 14.855 & -0.117 \\
\hline 0.03 & 1.204 & 0.410 & 0.206 & 14.612 & -0.110 \\
\hline 0.04 & 1.429 & 0.480 & 0.178 & 14.539 & -0.099 \\
\hline 0.05 & 1.687 & 0.611 & 0.122 & 15.574 & -0.063 \\
\hline 0.06 & 1.984 & 0.700 & 0.096 & 14.558 & -0.056 \\
\hline 0.08 & 2.453 & 0.857 & 0.053 & 14.293 & -0.038 \\
\hline 0.1 & 2.663 & 1.033 & 0.009 & 13.540 & -0.021 \\
\hline 0.15 & 2.745 & 1.280 & -0.043 & 11.334 & 0.006 \\
\hline 0.2 & 2.679 & 1.378 & -0.059 & 8.048 & 0.011 \\
\hline 0.3 & 2.482 & 1.468 & -0.078 & 16.930 & 0.014 \\
\hline 0.4 & 2.354 & 1.471 & -0.081 & 15.887 & 0.016 \\
\hline 0.5 & 2.250 & 1.454 & -0.080 & 16.301 & 0.018 \\
\hline 0.6 & 2.175 & 1.423 & -0.076 & 18.496 & 0.021 \\
\hline 0.8 & 2.051 & 1.384 & -0.073 & 15.311 & 0.021 \\
\hline 1 & 1.978 & 1.323 & -0.063 & 15.916 & 0.019 \\
\hline 1.5 & 1.864 & 1.232 & -0.048 & 14.873 & 0.017 \\
\hline 2 & 1.789 & 1.155 & -0.033 & 15.683 & 0.011 \\
\hline 3 & 1.678 & 1.061 & -0.012 & 14.248 & -0.001 \\
\hline 4 & 1.601 & 0.995 & 0.005 & 12.954 & -0.009 \\
\hline 5 & 1.537 & 0.947 & 0.021 & 12.308 & -0.021 \\
\hline 6 & 1.475 & 0.925 & 0.027 & 11.737 & -0.022 \\
\hline 8 & 1.405 & 0.902 & 0.034 & 13.708 & -0.027 \\
\hline 10 & 1.344 & 0.881 & 0.042 & 13.135 & -0.033 \\
\hline \multirow[t]{2}{*}{15} & 1.258 & 0.836 & 0.060 & 14.290 & -0.052 \\
\hline & \multicolumn{5}{|c|}{ FAB6 } \\
\hline 0.015 & 1.027 & 0.378 & 0.226 & 14.230 & -0.153 \\
\hline 0.02 & 1.058 & 0.406 & 0.199 & 15.816 & -0.114 \\
\hline 0.03 & 1.192 & 0.403 & 0.211 & 14.326 & -0.113 \\
\hline 0.04 & 1.403 & 0.468 & 0.184 & 14.417 & -0.103 \\
\hline 0.05 & 1.653 & 0.588 & 0.131 & 15.251 & -0.069 \\
\hline 0.06 & 1.907 & 0.702 & 0.093 & 15.187 & -0.052 \\
\hline 0.08 & 2.391 & 0.834 & 0.059 & 14.583 & -0.051 \\
\hline 0.1 & 2.607 & 1.010 & 0.014 & 13.694 & -0.031 \\
\hline 0.15 & 2.709 & 1.258 & -0.039 & 10.645 & -0.008 \\
\hline 0.2 & 2.654 & 1.361 & -0.055 & 8.052 & -0.006 \\
\hline 0.3 & 2.467 & 1.458 & -0.076 & 17.611 & 0.013 \\
\hline 0.4 & 2.344 & 1.461 & -0.079 & 16.295 & 0.015 \\
\hline 0.5 & 2.242 & 1.447 & -0.079 & 16.304 & 0.017 \\
\hline 0.6 & 2.165 & 1.421 & -0.076 & 17.812 & 0.020 \\
\hline 0.8 & 2.046 & 1.381 & -0.072 & 15.422 & 0.021 \\
\hline 1 & 1.972 & 1.323 & -0.063 & 16.288 & 0.019 \\
\hline 1.5 & 1.862 & 1.232 & -0.048 & 15.195 & 0.017 \\
\hline 2 & 1.788 & 1.155 & -0.033 & 15.931 & 0.011 \\
\hline 3 & 1.676 & 1.062 & -0.013 & 15.053 & -0.001 \\
\hline 4 & 1.600 & 0.996 & 0.005 & 12.975 & -0.009 \\
\hline 5 & 1.537 & 0.945 & 0.022 & 11.655 & -0.021 \\
\hline 6 & 1.478 & 0.928 & 0.026 & 11.817 & -0.021 \\
\hline 8 & 1.405 & 0.902 & 0.034 & 13.749 & -0.028 \\
\hline 10 & 1.344 & 0.883 & 0.042 & 13.122 & -0.033 \\
\hline 15 & 1.256 & 0.839 & 0.059 & 14.276 & -0.052 \\
\hline
\end{tabular}

materials such as concretes [14-16], gaseous mixture [17], human tissues [18], soils and ceramic [19, 20] which showed
TABLE 6: GP fitting parameters for FAB7 and FAB8.

\begin{tabular}{|c|c|c|c|c|c|}
\hline Energy $(\mathrm{MeV})$ & $b$ & $c$ & $a$ & $X_{K}$ & $d$ \\
\hline & \multicolumn{5}{|c|}{ FAB7 } \\
\hline 0.015 & 1.025 & 0.368 & 0.239 & 13.437 & -0.162 \\
\hline 0.02 & 1.055 & 0.416 & 0.189 & 16.700 & -0.111 \\
\hline 0.03 & 1.182 & 0.397 & 0.215 & 14.062 & -0.116 \\
\hline 0.04 & 1.381 & 0.459 & 0.188 & 14.336 & -0.107 \\
\hline 0.05 & 1.624 & 0.570 & 0.139 & 15.055 & -0.074 \\
\hline 0.06 & 1.863 & 0.687 & 0.098 & 15.178 & -0.054 \\
\hline 0.08 & 2.339 & 0.813 & 0.066 & 14.122 & -0.051 \\
\hline 0.1 & 2.554 & 0.989 & 0.019 & 13.808 & -0.038 \\
\hline 0.15 & 2.675 & 1.237 & -0.035 & 11.003 & -0.010 \\
\hline 0.2 & 2.630 & 1.345 & -0.053 & 8.423 & -0.007 \\
\hline 0.3 & 2.452 & 1.447 & -0.074 & 18.271 & 0.013 \\
\hline 0.4 & 2.336 & 1.452 & -0.077 & 16.691 & 0.015 \\
\hline 0.5 & 2.233 & 1.440 & -0.077 & 16.307 & 0.017 \\
\hline 0.6 & 2.157 & 1.419 & -0.075 & 17.148 & 0.019 \\
\hline 0.8 & 2.041 & 1.378 & -0.072 & 15.529 & 0.021 \\
\hline 1 & 1.965 & 1.323 & -0.063 & 16.649 & 0.020 \\
\hline 1.5 & 1.859 & 1.231 & -0.048 & 15.509 & 0.017 \\
\hline 2 & 1.787 & 1.155 & -0.033 & 16.068 & 0.011 \\
\hline 3 & 1.675 & 1.063 & -0.013 & 15.844 & -0.002 \\
\hline 4 & 1.599 & 0.996 & 0.005 & 12.995 & -0.009 \\
\hline 5 & 1.538 & 0.944 & 0.023 & 11.014 & -0.021 \\
\hline 6 & 1.480 & 0.930 & 0.025 & 11.897 & -0.020 \\
\hline 8 & 1.405 & 0.902 & 0.034 & 13.789 & -0.028 \\
\hline 10 & 1.343 & 0.884 & 0.041 & 13.110 & -0.033 \\
\hline \multirow[t]{2}{*}{15} & 1.255 & 0.842 & 0.058 & 14.263 & -0.052 \\
\hline & \multicolumn{5}{|c|}{ FAB8 } \\
\hline 0.015 & 1.024 & 0.358 & 0.251 & 12.694 & -0.171 \\
\hline 0.02 & 1.051 & 0.425 & 0.180 & 17.526 & -0.108 \\
\hline 0.03 & 1.173 & 0.395 & 0.215 & 14.061 & -0.116 \\
\hline 0.04 & 1.363 & 0.453 & 0.190 & 14.361 & -0.107 \\
\hline 0.05 & 1.597 & 0.555 & 0.146 & 14.946 & -0.079 \\
\hline 0.06 & 1.826 & 0.669 & 0.104 & 15.048 & -0.057 \\
\hline 0.08 & 2.071 & 0.792 & 0.073 & 13.676 & -0.051 \\
\hline 0.1 & 2.422 & 0.968 & 0.024 & 13.919 & -0.036 \\
\hline 0.15 & 2.642 & 1.217 & -0.031 & 11.354 & -0.012 \\
\hline 0.2 & 2.606 & 1.329 & -0.050 & 8.786 & -0.008 \\
\hline 0.3 & 2.437 & 1.437 & -0.073 & 18.919 & 0.013 \\
\hline 0.4 & 2.327 & 1.442 & -0.075 & 17.080 & 0.014 \\
\hline 0.5 & 2.225 & 1.434 & -0.076 & 16.310 & 0.016 \\
\hline 0.6 & 2.148 & 1.416 & -0.075 & 16.496 & 0.018 \\
\hline 0.8 & 2.035 & 1.375 & -0.071 & 15.634 & 0.021 \\
\hline 1 & 1.959 & 1.323 & -0.063 & 17.003 & 0.020 \\
\hline 1.5 & 1.857 & 1.230 & -0.048 & 15.819 & 0.017 \\
\hline 2 & 1.786 & 1.155 & -0.033 & 15.955 & 0.010 \\
\hline 3 & 1.674 & 1.064 & -0.013 & 16.350 & -0.002 \\
\hline 4 & 1.598 & 0.997 & 0.005 & 13.015 & -0.009 \\
\hline 5 & 1.538 & 0.942 & 0.024 & 10.380 & -0.022 \\
\hline 6 & 1.483 & 0.933 & 0.024 & 11.975 & -0.020 \\
\hline 8 & 1.405 & 0.902 & 0.034 & 13.828 & -0.028 \\
\hline 10 & 1.342 & 0.885 & 0.041 & 13.098 & -0.033 \\
\hline 15 & 1.254 & 0.845 & 0.058 & 14.249 & -0.051 \\
\hline
\end{tabular}

that the GP fitting is a very useful method for estimation of exposure and energy absorption build-up factors. Recently 
TABLE 7: GP fitting parameters for BOM and COB.

\begin{tabular}{|c|c|c|c|c|c|}
\hline Energy $(\mathrm{MeV})$ & $b$ & $c$ & $a$ & $X_{K}$ & $d$ \\
\hline & \multicolumn{5}{|c|}{ BOM } \\
\hline 0.015 & 1.020 & 0.379 & 0.231 & 11.998 & -0.148 \\
\hline 0.02 & 1.043 & 0.431 & 0.176 & 14.650 & -0.084 \\
\hline 0.03 & 1.143 & 0.394 & 0.213 & 14.390 & -0.115 \\
\hline 0.04 & 1.310 & 0.438 & 0.196 & 14.441 & -0.109 \\
\hline 0.05 & 1.519 & 0.515 & 0.164 & 14.678 & -0.091 \\
\hline 0.06 & 1.727 & 0.621 & 0.122 & 14.749 & -0.067 \\
\hline 0.08 & 2.135 & 0.759 & 0.081 & 13.507 & -0.050 \\
\hline 0.1 & 2.380 & 0.916 & 0.038 & 13.703 & -0.042 \\
\hline 0.15 & 2.561 & 1.171 & -0.022 & 12.064 & -0.016 \\
\hline 0.2 & 2.548 & 1.296 & -0.044 & 9.929 & -0.010 \\
\hline 0.3 & 2.419 & 1.397 & -0.064 & 13.335 & 0.001 \\
\hline 0.4 & 2.303 & 1.427 & -0.073 & 18.953 & 0.015 \\
\hline 0.5 & 2.211 & 1.423 & -0.074 & 16.293 & 0.015 \\
\hline 0.6 & 2.140 & 1.402 & -0.072 & 17.362 & 0.016 \\
\hline 0.8 & 2.032 & 1.362 & -0.068 & 16.187 & 0.018 \\
\hline 1 & 1.954 & 1.317 & -0.062 & 16.346 & 0.019 \\
\hline 1.5 & 1.846 & 1.230 & -0.048 & 15.414 & 0.016 \\
\hline 2 & 1.781 & 1.153 & -0.032 & 15.316 & 0.009 \\
\hline 3 & 1.673 & 1.058 & -0.011 & 11.803 & -0.002 \\
\hline 4 & 1.605 & 0.979 & 0.011 & 12.405 & -0.015 \\
\hline 5 & 1.531 & 0.953 & 0.019 & 10.723 & -0.017 \\
\hline 6 & 1.482 & 0.933 & 0.025 & 12.246 & -0.023 \\
\hline 8 & 1.398 & 0.913 & 0.031 & 13.872 & -0.027 \\
\hline 10 & 1.336 & 0.897 & 0.039 & 13.047 & -0.033 \\
\hline \multirow[t]{2}{*}{15} & 1.247 & 0.863 & 0.052 & 14.713 & -0.049 \\
\hline & \multicolumn{5}{|c|}{ COB } \\
\hline 0.015 & 1.017 & 0.296 & 0.321 & 10.490 & -0.248 \\
\hline 0.02 & 1.038 & 0.337 & 0.231 & 26.819 & -0.340 \\
\hline 0.03 & 1.106 & 0.376 & 0.225 & 13.775 & -0.048 \\
\hline 0.04 & 1.230 & 0.409 & 0.208 & 14.579 & -0.116 \\
\hline 0.05 & 1.388 & 0.465 & 0.187 & 14.455 & -0.105 \\
\hline 0.06 & 1.553 & 0.545 & 0.153 & 14.502 & -0.085 \\
\hline 0.08 & 1.846 & 0.706 & 0.093 & 14.638 & -0.054 \\
\hline 0.1 & 2.089 & 0.837 & 0.056 & 13.860 & -0.042 \\
\hline 0.15 & 2.342 & 1.074 & -0.003 & 13.216 & -0.023 \\
\hline 0.2 & 2.398 & 1.206 & -0.027 & 11.700 & -0.017 \\
\hline 0.3 & 2.330 & 1.327 & -0.050 & 8.675 & -0.010 \\
\hline 0.4 & 2.247 & 1.361 & -0.058 & 11.468 & -0.003 \\
\hline 0.5 & 2.159 & 1.387 & -0.068 & 20.771 & 0.017 \\
\hline 0.6 & 2.099 & 1.374 & -0.067 & 18.708 & 0.015 \\
\hline 0.8 & 2.002 & 1.344 & -0.064 & 16.552 & 0.017 \\
\hline 1 & 1.931 & 1.305 & -0.059 & 15.991 & 0.014 \\
\hline 1.5 & 1.834 & 1.224 & -0.047 & 15.476 & 0.015 \\
\hline 2 & 1.771 & 1.155 & -0.033 & 14.576 & 0.010 \\
\hline 3 & 1.670 & 1.058 & -0.010 & 10.534 & -0.003 \\
\hline 4 & 1.593 & 0.997 & 0.006 & 14.562 & -0.014 \\
\hline 5 & 1.525 & 0.963 & 0.017 & 10.978 & -0.016 \\
\hline 6 & 1.475 & 0.945 & 0.022 & 13.346 & -0.021 \\
\hline 8 & 1.392 & 0.924 & 0.028 & 13.653 & -0.025 \\
\hline 10 & 1.332 & 0.905 & 0.037 & 13.208 & -0.032 \\
\hline 15 & 1.240 & 0.878 & 0.048 & 14.114 & -0.045 \\
\hline
\end{tabular}

the radiation shielding by fly-ash concretes [14] and building materials [21] has been reported.
The innovative bricks using the residual fly-ash are considered high-quality building materials by the manufacturers which will potentially decrease some of the negative environmental impact of coal-fired power generation while meeting increasing demands for greener building materials $[22,23]$. Fly-ash brick (FAB), an environment-friendly costsaving building product, is an alternative to burnt clay bricks. The FAB is approximately stronger than common bricks with consistent strength. The FABs are ideally suited for internal, external, load bearing, and nonload bearing walls. FABs are durable, economical, and eco-friendly and have low water absorption (8-12\%), less mortar consumption, and low energy consumption with the lowest green house impact. These bricks are not affected by environmental conditions and remain static thus ensuring longer life of the building. These bricks are economical/cost-effective and nil wastage while transporting and handling. The houses and buildings in which people are living are constructed by the bricks made up of soil and environmental-friendly fly-ashes. The potential applications of fly-ash are shielding materials [24], glasses $[25,26], \mathrm{X}$-ray shielding [27], electromagnetic radiation in Xband and Ku-band shielding [28], and houses and building construction $[22,23]$.

In view of radiation safety inside the houses or buildings constructed by FAB, a theoretical gamma-ray exposure buildup factor (EBF) and fast neutron removal cross section have been calculated. We have calculated the EBF of eight types of FABs, brick of mud (BOM), and common brick (COB) by GP fitting in the photon energy range from 0.015 to $15 \mathrm{MeV}$ up to $40 \mathrm{mfp}$. Comparative analysis shows that higher buildup factors exist for FABs and lower fast neutron removal cross sections. High EBF proves that FABs are poor gamma shielding for the construction of houses and buildings. The study reveals that brick of mud and common brick are lowcost safe building materials against radiation. It should be noted that this study is valuable in shielding analysis and estimation of emergency dose.

\section{Computation Method}

The elemental compositions of the fly-ash bricks are given in Table 1 [1]. These fly-ash bricks samples are prepared by a formula (Lime) $)_{0.15}$ (Gypsum $)_{0.05}$ (Fly Ash) $\times(\text { Soil })_{0.8-x}$, where the values of $x$ range from 0.4 to 0.7 . Two other bricks of mud (BOM) and common brick (COM) are also analyzed for comparison of shielding properties for gamma-ray and neutron. The build-up calculation by GP fitting method and fast neutron removal cross section is explained below.

2.1. Exposure Build-Up Factor. The EBF of the bricks and the GP fitting parameters are calculated by method of interpolation from the equivalent atomic number, $Z_{\text {eq }}$, of the bricks. The computational work of these parameters is done in three steps as follows:

(1) calculation of equivalent atomic number (Table 2),

(2) calculation of GP fitting parameters (Tables 3, 4, 5, 6, and 7),

(3) calculation of build-up factors. 
TABLE 8: Fast neutron removal cross section of fly-ash brick and other bricks.

(a)

\begin{tabular}{|c|c|c|c|c|c|c|c|c|c|c|}
\hline \multirow[t]{2}{*}{ Ele. } & $\begin{array}{r}\text { FA } \\
\rho=1.87\end{array}$ & $\begin{array}{l}\mathrm{AB1} \\
\mathrm{g} \mathrm{cm}\end{array}$ & \multicolumn{2}{|c|}{$\begin{array}{c}\text { FAB2 } \\
\rho=1.81 \mathrm{~g} \mathrm{~cm}^{-3}\end{array}$} & \multicolumn{2}{|c|}{$\begin{array}{c}\text { FAB } \\
\rho=1.76 \mathrm{~g} \mathrm{~cm}^{-3}\end{array}$} & \multicolumn{2}{|c|}{$\begin{array}{c}\text { FAB } 4 \\
\rho=1.72 \mathrm{~g} \mathrm{~cm}^{-3}\end{array}$} & \multicolumn{2}{|c|}{$\begin{array}{c}\text { FAB5 } \\
\rho=1.85 \mathrm{~g} \mathrm{~cm}^{-3}\end{array}$} \\
\hline & $\begin{array}{l}\text { Partial } \\
\text { density }\end{array}$ & $\Sigma_{R}\left(\mathrm{~cm}^{-1}\right)$ & $\begin{array}{l}\text { Partial } \\
\text { density }\end{array}$ & $\Sigma_{R}\left(\mathrm{~cm}^{-1}\right)$ & $\begin{array}{l}\text { Partial } \\
\text { density }\end{array}$ & $\Sigma_{R}\left(\mathrm{~cm}^{-1}\right)$ & $\begin{array}{l}\text { Partial } \\
\text { density }\end{array}$ & $\Sigma_{R}\left(\mathrm{~cm}^{-1}\right)$ & $\begin{array}{l}\text { Partial } \\
\text { density }\end{array}$ & $\Sigma_{R}\left(\mathrm{~cm}^{-1}\right)$ \\
\hline $\mathrm{H}$ & $1.68 E-03$ & $1.01 E-03$ & $1.63 E-03$ & $9.74 E-04$ & $1.58 E-03$ & $9.47 E-04$ & $1.55 E-03$ & $9.26 E-04$ & $1.67 E-03$ & $9.96 E-04$ \\
\hline $\mathrm{C}$ & $6.90 E-02$ & $3.46 E-03$ & $6.17 E-02$ & $3.10 E-03$ & $5.51 E-02$ & $2.77 E-03$ & $4.88 E-02$ & $2.45 E-03$ & $3.15 E-02$ & $1.58 E-03$ \\
\hline $\mathrm{N}$ & $0.00 E+00$ & $0.00 E+00$ & $0.00 E+00$ & $0.00 E+00$ & $0.00 E+00$ & $0.00 E+00$ & $0.00 E+00$ & $0.00 E+00$ & $0.00 E+00$ & $0.00 E+00$ \\
\hline $\mathrm{O}$ & $9.11 E-01$ & $3.69 E-02$ & $8.81 E-01$ & $3.57 E-02$ & $8.57 E-01$ & $3.47 E-02$ & $8.37 E-01$ & $3.39 E-02$ & $9.24 E-01$ & $3.74 E-02$ \\
\hline $\mathrm{Na}$ & $1.87 E-04$ & $6.38 E-06$ & $1.81 E-04$ & $6.17 E-06$ & $1.76 E-04$ & $6.00 E-06$ & $1.72 E-04$ & $5.87 E-06$ & $2.04 E-03$ & $6.94 E-05$ \\
\hline $\mathrm{Mg}$ & $2.00 E-02$ & $6.66 E-04$ & $1.86 E-02$ & & $1.74 E-02$ & & $1.62 E-02$ & $5.38 E-04$ & $1.42 E-02$ & $4.74 E-04$ \\
\hline $\mathrm{Al}$ & $2.20 E-01$ & $6.45 E-03$ & $2.01 E-01$ & $5.90 E-03$ & $1.85 E-01$ & $5.42 E-03$ & $1.70 E-01$ & $4.97 E-03$ & $1.99 E-01$ & $5.84 E-03$ \\
\hline $\mathrm{Si}$ & $4.34 E-01$ & $1.28 E-02$ & $4.18 E-01$ & $1.23 E-02$ & $4.04 E-01$ & $1.19 E-02$ & $3.93 E-01$ & $1.16 E-02$ & $4.64 E-01$ & $1.37 E-02$ \\
\hline $\mathrm{P}$ & $0.00 E+00$ & $0.00 E+00$ & $0.00 E+00$ & $0.00 E+00$ & $0.00 E+00$ & $0.00 E+00$ & $0.00 E+00$ & $0.00 E+00$ & $1.30 E-03$ & $3.66 E-05$ \\
\hline S & $7.48 E-04$ & $2.07 E-05$ & $7.24 E-04$ & $2.01 E-05$ & $5.28 E-04$ & $1.46 E-05$ & $5.16 E-04$ & $1.43 E-05$ & $3.70 E-04$ & $1.02 E-05$ \\
\hline K & $7.85 E-03$ & $1.94 E-04$ & $1.45 E-02$ & $3.58 E-04$ & $2.08 E-02$ & $5.13 E-04$ & $2.68 E-02$ & $6.63 E-04$ & $8.14 E-03$ & $2.01 E-04$ \\
\hline $\mathrm{Ca}$ & $1.29 E-01$ & $3.15 E-03$ & $1.23 E-01$ & $2.98 E-03$ & $1.16 E-01$ & $2.83 E-03$ & $1.11 E-01$ & $2.70 E-03$ & $1.35 E-01$ & $3.29 E-03$ \\
\hline $\mathrm{Ti}$ & $1.87 E-04$ & $3.83 E-06$ & $1.81 E-04$ & $3.71 E-06$ & $1.76 E-04$ & $3.61 E-06$ & $1.72 E-04$ & $3.53 E-06$ & $1.05 E-02$ & $2.16 E-04$ \\
\hline $\mathrm{Mn}$ & $1.87 E-04$ & $3.80 E-06$ & $1.81 E-04$ & $3.67 E-06$ & $1.76 E-04$ & $3.57 E-06$ & $1.72 E-04$ & $3.49 E-06$ & $1.85 E-04$ & $3.76 E-06$ \\
\hline $\mathrm{Fe}$ & $7.61 E-02$ & $1.63 E-03$ & $8.96 E-02$ & $1.92 E-03$ & $1.03 E-01$ & $2.20 E-03$ & $1.16 E-01$ & $2.48 E-03$ & $5.77 E-02$ & $1.24 E-03$ \\
\hline Total & 1.87 & 0.0663 & 1.81 & 0.0639 & 1.76 & 0.0619 & 1.72 & 0.0602 & 1.85 & 0.0651 \\
\hline
\end{tabular}

(b)

\begin{tabular}{|c|c|c|c|c|c|c|c|c|c|c|}
\hline \multirow[t]{2}{*}{ Ele. } & \multicolumn{2}{|c|}{$\begin{array}{l}\text { FAB6 } \\
1.79 \mathrm{~g} \mathrm{~cm}^{-3}\end{array}$} & \multicolumn{2}{|c|}{$\begin{array}{c}\text { FAB7 } \\
\rho=1.74 \mathrm{~g} \mathrm{~cm}^{-3}\end{array}$} & \multicolumn{2}{|c|}{$\begin{array}{c}\text { FAB8 } \\
\rho=1.70 \mathrm{~g} \mathrm{~cm}^{-3}\end{array}$} & \multicolumn{2}{|c|}{$\begin{array}{c}\text { BOM } \\
\rho=1.82 \mathrm{~g} \mathrm{~cm}^{-3}\end{array}$} & \multicolumn{2}{|c|}{$\begin{array}{c}\text { COB } \\
\rho=1.87 \mathrm{~g} \mathrm{~cm}^{-3}\end{array}$} \\
\hline & $\begin{array}{l}\text { artial } \\
\text { ensity }\end{array}$ & $\Sigma_{R}\left(\mathrm{~cm}^{-1}\right)$ & $\begin{array}{l}\text { Partial } \\
\text { density }\end{array}$ & & $\begin{array}{l}\text { Partial } \\
\text { density }\end{array}$ & & & & & \\
\hline $\mathrm{H}$ & & & & & & & & & & \\
\hline 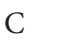 & & & $6 E-02$ & $8 E-03$ & & & & & & \\
\hline $\mathrm{N}$ & $0 E+00$ & $.00 E+00$ & $.00 E+00$ & $0.00 E+00$ & $00 E+00$ & $.00 E+00$ & $00 E+00$ & & $37 E-04$ & $8.38 E-06$ \\
\hline $\mathrm{O}$ & $1 E-01$ & $3.61 E-02$ & $8.62 E-01$ & $3.49 E-02$ & $.39 E-01$ & $3.40 E-02$ & $9.93 E-01$ & & $8.48 E-01$ & $3.43 E-02$ \\
\hline & & & & & & & & & & \\
\hline & & & & & & & & & & -05 \\
\hline & $E-01$ & $39 E-03$ & $E-01$ & 4.9 & & & & & -01 & -03 \\
\hline $\mathrm{Si}$ & $2 E-01$ & $1.30 E-02$ & $E-01$ & $1.25 E-02$ & $6 E-01$ & & $8 E-01$ & & -01 & -02 \\
\hline $\mathrm{P}$ & $7 E-03$ & $.04 E-05$ & $8.70 E-04$ & $2.46 E-05$ & $6.80 E-04$ & & $0.00 E+00$ & & $0.00 E+00$ & $0.00 E+00$ \\
\hline S & $58 E-04$ & & $3.48 E-04$ & $9.64 E-06$ & & & & & & $1.04 E-05$ \\
\hline K & $1.47 E-02$ & & & & & & & & $4.97 E-02$ & $1.23 E-03$ \\
\hline $\mathrm{Ca}$ & $7 E-01$ & & & $2.91 E-03$ & & & & & -02 & $6 E-03$ \\
\hline $\mathrm{Ti}$ & $77 E-03$ & & & & & & $0.00 E+00$ & & $1.68 E-03$ & $3.45 E-05$ \\
\hline & $79 E-04$ & $3.63 E-06$ & $1.74 E-04$ & $3.53 E-06$ & $1.70 E-04$ & & $0.00 E+00$ & & $3.18 E-03$ & $6.45 E-05$ \\
\hline $\mathrm{Fe}$ & $43 E-02$ & $1.59 E-03$ & $8.99 E-02$ & $1.92 E-03$ & $1.05 E-01$ & $2.25 E-03$ & $5.46 E-03$ & $1.17 E-04$ & $3.36 E-01$ & $7.19 E-03$ \\
\hline tal & 1.79 & 0.0628 & 1.74 & 0.0608 & 1.70 & 0.0593 & 1.82 & 0.0614 & 1.87 & 0.0636 \\
\hline
\end{tabular}

$Z_{\text {eq }}$ is a parameter which describes the material properties in terms of equivalent elements similar to atomic number for a single element. Since interaction processes of gammaray photon with matter, photo-electric absorption, Compton scattering, and pair-production are energy dependent, therefore $Z_{\mathrm{eq}}$ for each interaction varies according to the photon energy. However the build-up of photons in the medium is mainly due to multiple scattering events by Compton scattering, so that $Z_{\mathrm{eq}}$ is derived from the Compton scattering interaction process.

The $Z_{\text {eq }}$, for individual brick, is estimated by the ratio of $(\mu / \rho)_{\text {Compton }} /(\mu / \rho)_{\text {Total }}$, at a specific energy with the 


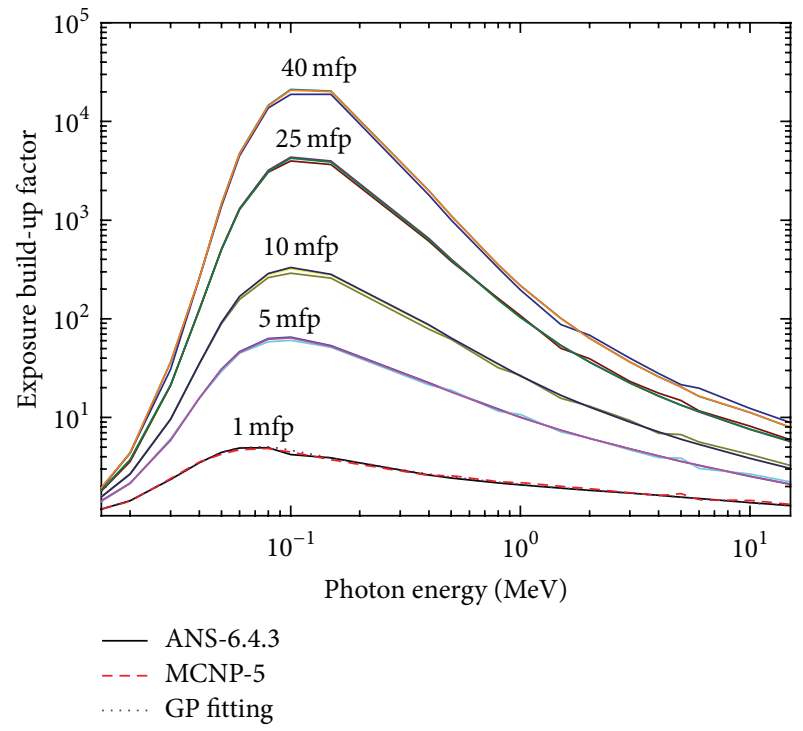

FIGURE 1: Exposure build-up factors for water by ANS-6.4.3, MCNP5 , and GP fitting.

correspondance of an element at the same energy. Thus first the Compton partial mass attenuation coefficient, $(\mu / \rho)_{\text {Compton, and the total mass attenuation coefficients, }}$ $(\mu / \rho)_{\text {Total }}$, are obtained for elements $Z=4$ to 40 for the selected bricks in the energy region from 0.0015 to $15 \mathrm{MeV}$ using WinXCom $[29,30]$.

The interpolation of $Z_{\mathrm{eq}}$ is employed by the following formula [31, 32]:

$$
Z_{\text {eq }}=\frac{Z_{1}\left(\log R_{2}-\log R_{1}\right)+Z_{2}\left(\log R-\log R_{1}\right)}{\log R_{2}-\log R_{1}},
$$

where $Z_{1}$ and $Z_{2}$ are the atomic numbers of the elements corresponding to the ratios $R_{1}$ and $R_{2}$, respectively. $R$ is the ratio, $(\mu / \rho)_{\text {Compton }} /(\mu / \rho)_{\text {Total }}$, at specific energy and the ratio $(\mu / \rho)_{\text {Compton }} /(\mu / \rho)_{\text {Total }}$ for $Z_{\text {eq }}$ lies between two successive ratios of the elements.

The GP fitting parameters are calculated in a similar fashion of interpolation procedure for $Z_{\text {eq }}$. The GP fitting parameters for the elements were taken from the ANS6.4.3 standard reference database which provides the GP fitting parameters for twenty three elements ( $Z=4$ to 92) in the energy region from 0.015 to $15 \mathrm{MeV}$ up to $40 \mathrm{mfp}$ penetration depth. The GP fitting parameters for the bricks were interpolated using a similar formula:

$$
C=\frac{C_{1}\left(\log Z_{2}-\log Z_{\mathrm{eq}}\right)+C_{2}\left(\log Z_{\mathrm{eq}}-\log Z_{1}\right)}{\log Z_{2}-\log Z_{1}}
$$

where $C_{1}$ and $C_{2}$ are the values of the GP fitting parameters corresponding to the atomic numbers of $Z_{1}$ and $Z_{2}$, respectively, at a given energy.

The third and final step is build-up factors estimation by GP fitting parameters $\left(b, c, a, X_{K}\right.$, and $\left.d\right)$ in the photon energy range of $0.015-15 \mathrm{MeV}$ up to a $40 \mathrm{mfp}$ by the following equations [13]:

$$
\begin{gathered}
B(E, X)=1+\frac{b-1}{K-1}\left(K^{x}-1\right) \quad \text { for } K \neq 1, \\
B(E, X)=1+(b-1) X \quad \text { for } K=1, \\
K(E, X)=C X^{a}+d \frac{\tanh \left(X / X_{K}-2\right)-\tanh (-2)}{1-\tanh (-2)}
\end{gathered}
$$

for penetration depth $(X) \leq 40 \mathrm{mfp}$,

where $X$ is the source-detector distance for the medium in terms of $\mathrm{mfp}$ and $b$ the value of the exposure build-up factor at $1 \mathrm{mfp}, K(E, X)$ is the dose multiplicative factor, and $b, c, a$, $X_{K}$, and $d$ are computed GP fitting parameters which depend on the attenuating medium and source energy.

2.2. Fast Neutron Removal Cross Section. An approximate method for calculation of the attenuation of fast neutrons by use of an effective removal cross section has been developed to allow for scattering or build-up. The effective removal crosssection for compounds and homogenous mixtures may be calculated from the value of $\Sigma_{R}\left(\mathrm{~cm}^{-1}\right)$ or $\Sigma_{R} / \rho\left(\mathrm{cm}^{2} / \mathrm{g}\right)$ for various elements in the compounds by mixture rule. Difference in application of mixture for neutron interaction differs as weight fraction is replaced by partial density and mass attenuation coefficient by neutron removal cross section:

$$
\begin{aligned}
& \frac{\Sigma_{R}}{\rho}=\sum_{i} w_{i}\left(\frac{\Sigma_{R}}{\rho}\right)_{i}, \\
& \frac{\Sigma_{R}}{\rho}=\sum_{i} \rho_{i}\left(\frac{\Sigma_{R}}{\rho}\right)_{i} .
\end{aligned}
$$

The values obtained for effective neutron removal cross section by the above equations are accurate within $10 \%$ of the experimental values investigated for aluminium, beryllium, graphite, hydrogen, iron, lead, oxygen, boron carbide, and so forth [33]. The $\Sigma_{R} / \rho$ values of elements of the bricks have been taken from Kaplan and Chilton et al. [34, 35].

\section{Uncertainties}

The uncertainties in the build-up factor estimated by GP fitting are comparable with ANS-6.4.3 standard and MCNP-5 [36] for air and water. Figure 1 shows that the EBFs in water by GP fitting, ANS-6.4.3 standard, and MCNP-5 at different photon energies are comparable. The MCNP-5 results vary from those ANS-6.4.3 standards with greatest $13.83 \%$ due to difference in cross section libraries, method of solution for codes, calculation methods, standard deviation, and physics assumptions for bremsstrahlung and coherent scattering [36]. It is found that the invariant embedding, GP fitting, and MCNP simulation are in good agreement with 18 low- $Z$ materials with small discrepancies [12]. This quantitative and comparative approach shows that our results for gamma-ray EBF will be of small uncertainties for easy establishment of the data and analysis by GP fitting methodology. 

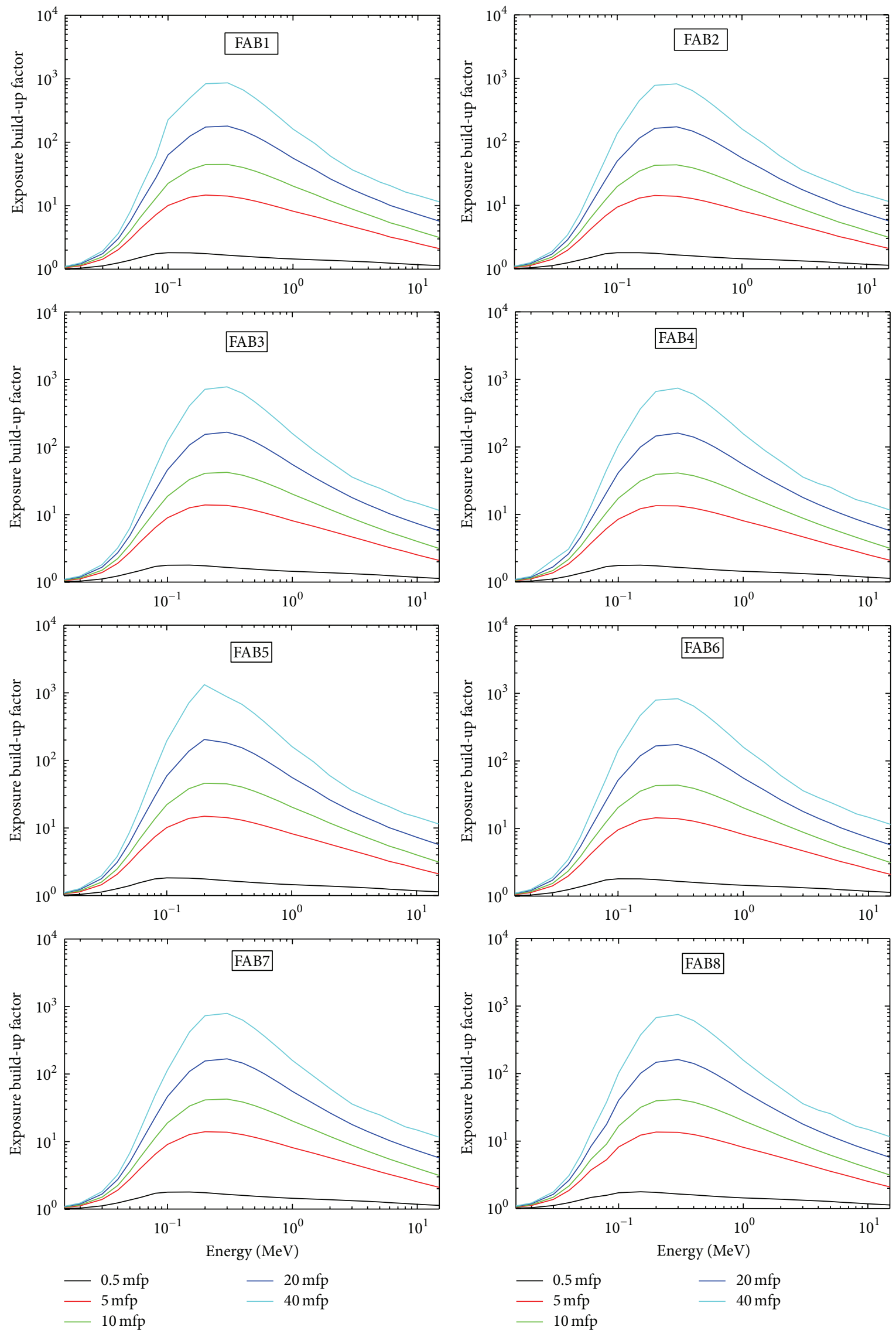

FIgURE 2: Continued. 

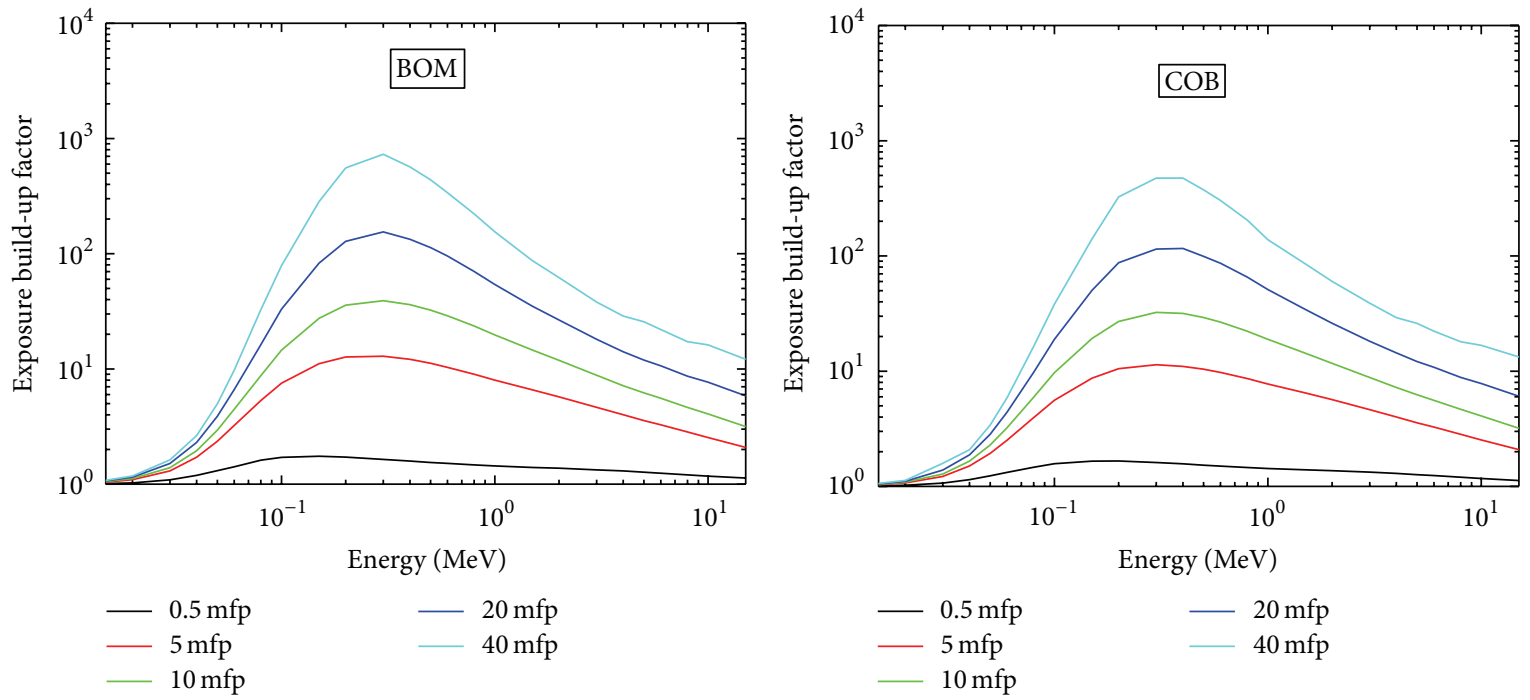

FIGURE 2: Variation of exposure build-up factor of fly-ash bricks, brick of mud, and common brick with incident photon energy.

\section{Result and Discussion}

The photon energy dependency of the EBFs is shown in Figure 2 at various penetration depths $(0.5,5,10,20,30$, and $40 \mathrm{mfp}$ ) for each FAB, BOM, and COB. Variation of EBF with penetration depths is shown in Figure 3 at photon energies $0.015,0.15,1.5$, and $15 \mathrm{MeV}$. The chemical compositions of the selected materials are investigated at penetration depths of $0.5,5,10,20,30$, and $40 \mathrm{mfp}$ and shown in Figure 4 . The fast neutron removal cross sections of the studies bricks are given in Tables $8(\mathrm{a})$ and $8(\mathrm{~b})$. The variation of EBF with photon energy, penetration depth, and chemical compositions is explained in next coming sections.

4.1. Photon Energy Dependency of Exposure Build-Up Factor. The variation of EBF values of the $\mathrm{FAB}, \mathrm{BOM}$, and $\mathrm{COB}$ with photon energy is shown in Figure 2. It is noted that the EBF values of the bricks are minimum in low-energy and high-energy photons whereas they are higher in the intermediate-energy photons. The build-up factor in lowenergy is small because the photons are completely absorbed by photo-electric absorption, gradually increases with the energy due to multiple scattering by the Compton scattering in the intermediate-energy region and finally again reduces in high-energy region due to pair-production. The analysis shows that the brick having, lowest EBF should be considered for gamma-ray shielding. The gamma build-up factors are the lowest for $0.5 \mathrm{mfp}$, and highest for $40 \mathrm{mfp}$, due to multiple scattering events for large penetration depth of $40 \mathrm{mfp}$. The EBFs of the FAB, BOM, and $\mathrm{COB}$ are found higher than the ordinary concretes [16].

4.2. Penetration Depth Dependency of Exposure Build-Up Factor. The variation of EBFs of the selected FABs, BOM, and $\mathrm{COB}$ with penetration depths at photon energies 0.015 , $1.5,5$, and $15 \mathrm{MeV}$ is shown in Figure 3. It is observed that the EBF values increase with the penetration depth initially and afterwards become stable. The EBFs for the BOM and $\mathrm{COB}$ are found lesser than FABs at photon energies 0.15 , 0.15 , and $1.5 \mathrm{MeV}$ photon energy. It can be seen that the values of EBF of the bricks increase with increase in $Z_{\text {eq }}$ at $15 \mathrm{MeV}$ as penetration depth increases. In the low photon energy region $(\sim 1.5 \mathrm{MeV})$, exposure build-up factor becomes independent of penetration depth above $25 \mathrm{mfp}$. The reason may be that beyond $3 \mathrm{MeV}$, the pair-production dominates the Compton scattering and produces the electron-positron pairs. These particles may escape from the medium of lower penetration depth whereas they will scatter in large penetration depths as well as originate the secondary gamma photon by annihilation to increase the gamma photon intensities. The smallest value of EBFs of COB for low energy $(<1.22 \mathrm{MeV})$ gradually increases with increase in photon energy due pairproduction.

4.3. Chemical Composition Dependency of Exposure BuildUp Factor. The EBFs are dependent on the atomic number of elements; hence EBF values of the materials are dependent on chemical compositions [16]. The chemical element composition dependency on the EBF is analyzed by for the photon energy at constant penetration depths as shown in Figure 4. It is observed that the EBF values are small at low- as well as high-energy photons whereas maximum in the intermediate-energy photons. The reason for peaked EBF values is that the photo-electric and pair-production are dominant interaction processes in low- and high-energy, respectively, which completely remove the photons. The intermediate-energy photon takes over Compton scattering where photon goes under multiple scattering events to buildup the exposure. The brick materials having large weight fraction of low- $Z$ elements are showing high EBF values due to less removal of the photons.

The fly-ash brick (low $Z_{\text {eq }}$ ) is having larger EBF compared with other bricks (higher $Z_{\mathrm{eq}}$ ) for entire energy region from 0.015 to $15 \mathrm{MeV}$ in low penetration depths. The EBF of 


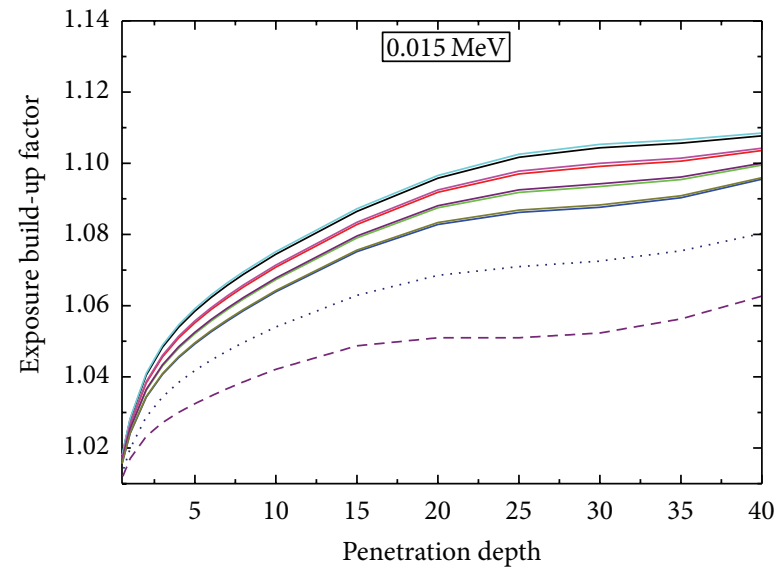

(a)

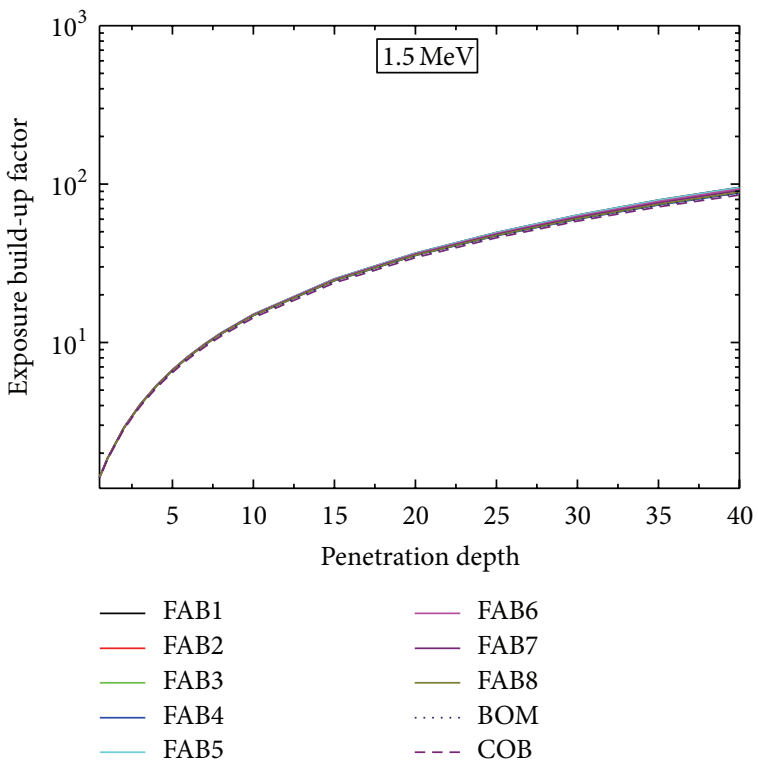

(c)

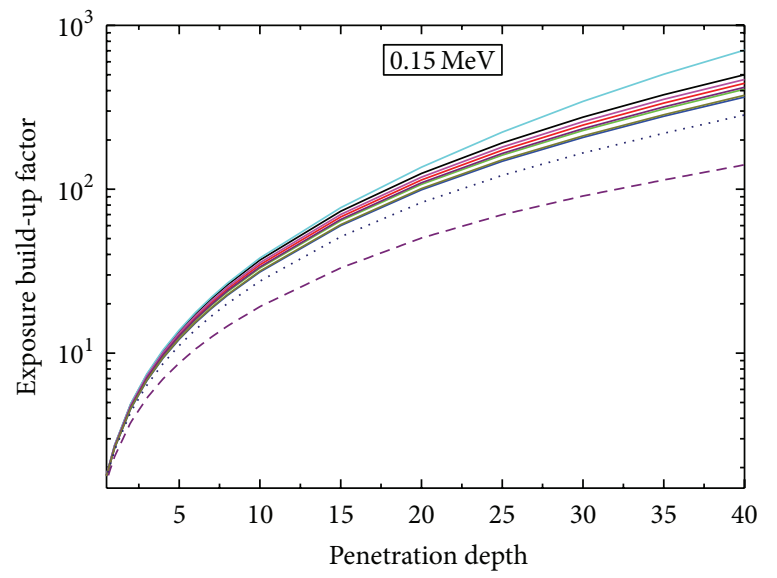

(b)

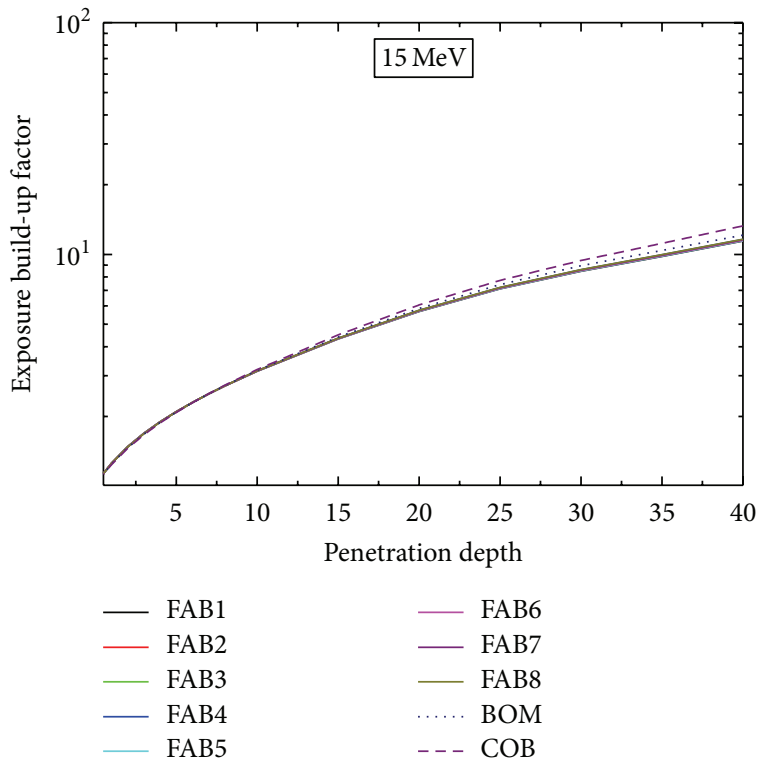

(d)

FIGURE 3: Variation of exposure build-up factor of fly-ash bricks, brick of mud, and common brick with penetration depth for photon energies $0.015,0.15,1.5$, and $15 \mathrm{MeV}$.

$\mathrm{BOM}$ and $\mathrm{COB}$ shows behavior in reverse order at $3 \mathrm{Mev}$ for large penetration depth. Above $3 \mathrm{MeV}$ photon energy and for large penetration depths (above $10 \mathrm{mfp}$ ), exposure build-up factor becomes directly proportional to $Z_{\text {eq. }}$. It may be due to dominance of pair-production above $1.22 \mathrm{MeV}$ which increases with increase in photon energy and results in production of electron and positron. The positron annihilates at zero kinetic energy and produces new two photons of energy $0.511 \mathrm{MeV}$ which build-up in the medium due to multiple scattering events. The pair-production dependent on $Z^{2}$ signifies that the $\mathrm{COB}$ and $\mathrm{BOM}$ should have large photon intensities of energies $0.511 \mathrm{MeV}$. The EBF is found large in the higher penetration depths due to possibilities of multiple scattering whereas the positron may escape though the low penetration depth.
4.4. Fast Neutron Removal Cross Section. In case of neutron attenuation, the effective fast neutron removal (FNR) cross sections of the different $\mathrm{FAB}, \mathrm{BOM}$, and $\mathrm{COB}$ are given in Tables $8(\mathrm{a})$ and $8(\mathrm{~b})$. The effective removal cross section is approximately constant for neutron energy from 2 to $12 \mathrm{MeV}$ [34]. In Tables $8(\mathrm{a})$ and $8(\mathrm{~b})$, the calculations are being listed for various elemental compositions of the $\mathrm{FAB}$ and other bricks. The effective FNR cross section $\Sigma_{R}\left(\mathrm{~cm}^{-1}\right)$ of the selected brick materials is calculated by partial density of each element and FNR cross section. It is observed that the removal cross section is higher for FAB1, and this leads to the conclusion that the FAB1 is more effective for neutron attenuation than the FABs, BOM, and COB. Also, from point of neutron shielding, fly-ash and common brick materials are more suitable replacement of brick of mud. The low 


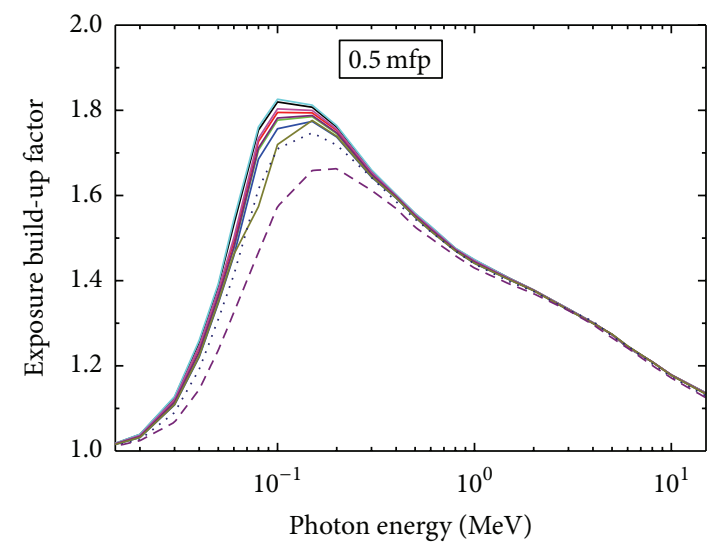

(a)

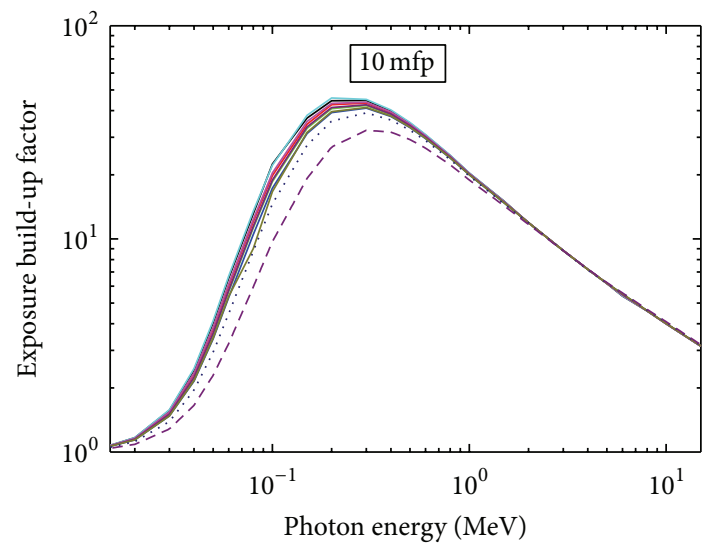

(c)

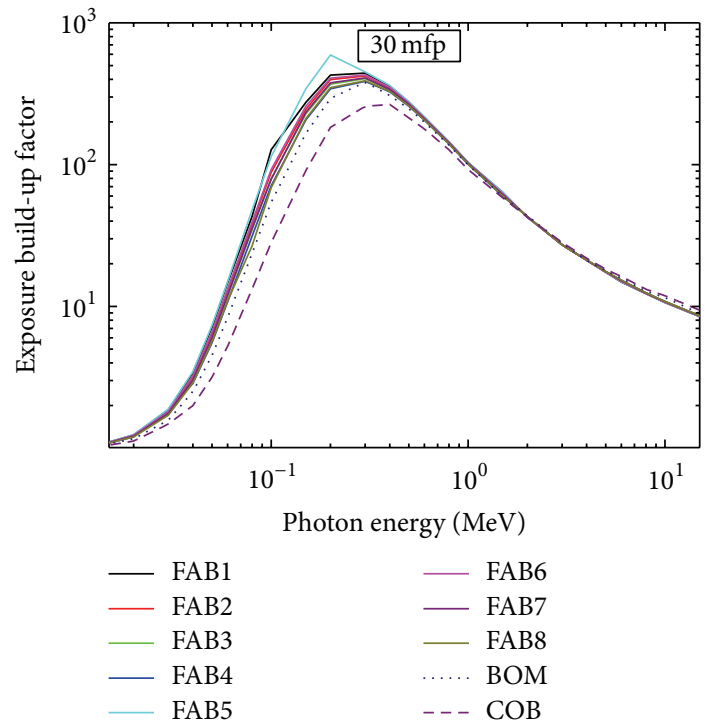

(e)

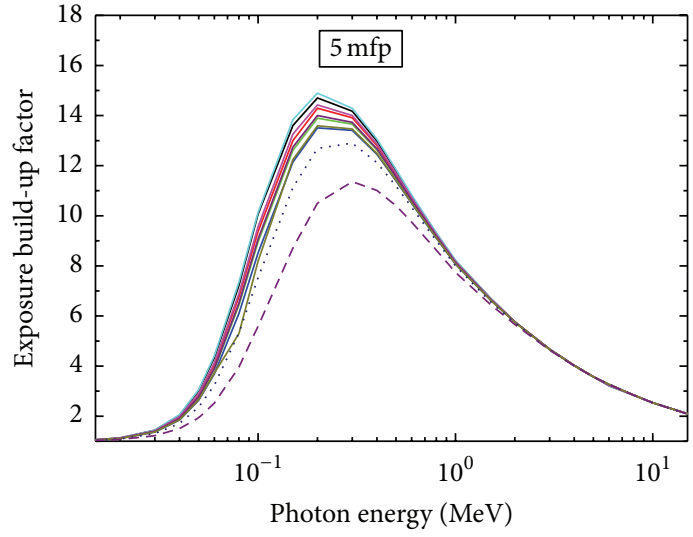

(b)

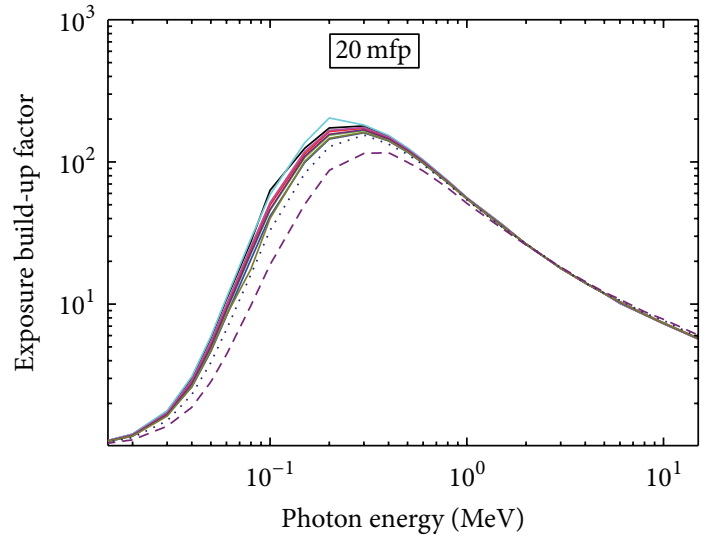

(d)
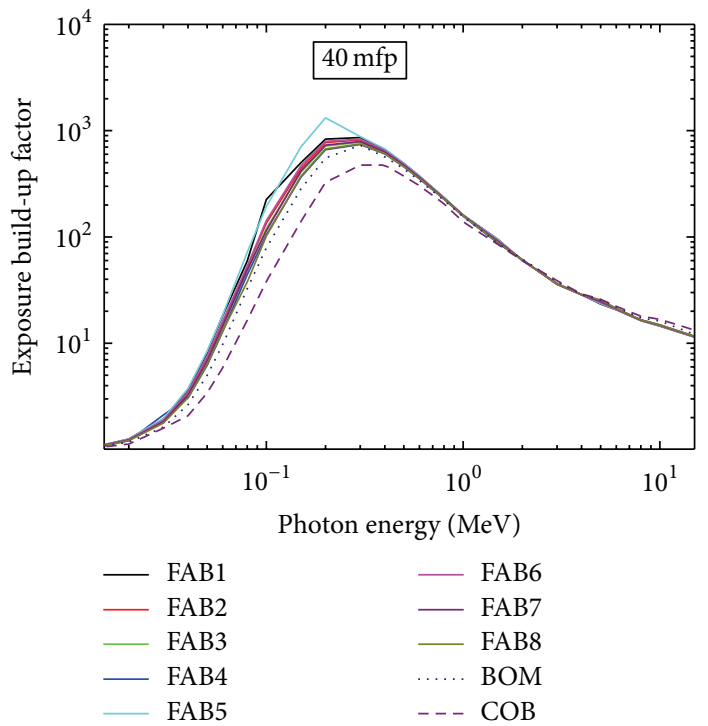

(f)

FIGURE 4: Variation of exposure build-up factor of fly-ash bricks, brick of mud, and common brick with photon energy for penetration depths $0.5,5,10,20,30$, and $40 \mathrm{mfp}$. 
variation in removal cross section is being noticed for all types of the bricks. Elevated values of removal cross section of FAB1 and $\mathrm{COB}$ are attributed due to high density and large contribution of low atomic number weight fractions of $\mathrm{H}$ and $\mathrm{C}$. With constant $\mathrm{H}$ weight fraction and variable densities, the removal cross section varies. In cases of $\mathrm{COB}$, the removal cross section is slightly higher due to large $\mathrm{C}$ weight fraction with equal value of density of FAB1. Therefore it is concluded that low atomic number elemental composition and density contribute a vital role in gamma-ray and neutron shielding properties.

\section{Conclusions}

In the present studies, we have calculated the exposure buildup factors of fly-ash bricks for photon energies from 0.015 to $15 \mathrm{MeV}$ up to $40 \mathrm{mfp}$ penetration depth by geometrical progression (GP) method. Exposure build-up factor increases with the increase in penetration depth for all the fly-ash bricks. For the entire energy region (0.015-15.0 MeV), in case of small penetration depths (below $10 \mathrm{mfp}$ ), exposure buildup factor is inversely proportional to the $Z_{\text {eq }}$. In the low photon energy region $(\approx 1.5 \mathrm{MeV})$, exposure build-up factor becomes dependent upon penetration depth above $25 \mathrm{mfp}$. Above $3 \mathrm{MeV}$ photon energy and for large penetration depths (above $10 \mathrm{mfp}$ ), exposure build-up factor becomes directly proportional to $Z_{\mathrm{eq}}$. Bricks containing low atomic number elements show high fast neutron removal cross. Low atomic number elemental composition and density contribute a vital role in gamma-ray and neutron shielding properties. The study should be very useful for shielding effectiveness of flyash bricks and dose estimation.

\section{Acknowledgments}

The authors are grateful to respected Dr. M. J. Berger and J. H. Hubbell and L. Gerward for providing the state-of-the-art and user-friendly computer programs XCOM/WinXCom.

\section{References}

[1] S. M. Kulwinder, K. Baljit, S. S. Gurdeep, and K. Ajay, "Investigations of some building materials for $\gamma$-rays shielding effectiveness," Radiation Physics and Chemistry, vol. 87, pp. 1625, 2013.

[2] G. R. White, "The penetration and diffusion of $\mathrm{Co}^{60}$ gammarays in water using spherical geometry," Physical Review, vol. 80, no. 2, pp. 154-156, 1950.

[3] U. Fano, "Gamma-ray attenuation-part II: analysis of penetration," Nucleonics, vol. 11, pp. 55-61, 1953.

[4] Y. Harima, "An historical review and current status of buildup factor calculations and applications," Radiation Physics and Chemistry, vol. 41, no. 4-5, pp. 631-672, 1993.

[5] K. Takeuchi and S. Tanaka, "PALLAS-ID (VII). A code for direct integration of transport equation in one-dimensional plane and spherical geometries," Tech. Rep. 84, Japan Atomic Energy Research Institute, 1984.

[6] G. L. Simmons, "An adjoint gamma-ray moments computer code, ADJMOM-I," NBS Technical Note 748, National Bureau of Standards, 1973.
[7] A. B. Chilton, C. M. Eisenhauer, and G. L. Simmons, "Photon point source buildup factors for air, water and iron," Nuclear Science and Engineering, vol. 73, pp. 97-107, 1980.

[8] D. V. Gopinath and K. Samthanam, "Radiation transport in one dimensional finite system-part I: development in anisotropic source flux technique," Nuclear Science and Engineering, vol. 43, pp. 186-196, 1971.

[9] W. R. Nelson, H. Hirayama, and D. W. O. Rogers, EGS4 Code System, SLAC-265, Stanford Linear Accelerator Centre, Stanford, Calif, USA, 1985.

[10] ANSI/ANS-6.4.3, "Gamma ray attenuation coefficient and buildup factors for engineering materials," 1991.

[11] A. Shimizu, "Calculation of gamma-ray buildup factors up to depths of $100 \mathrm{mfp}$ by the method of invariant embedding, (I): analysis of accuracy and comparison with other data," Journal of Nuclear Science and Technology, vol. 39, no. 5, pp. 477-486, 2002.

[12] A. Shimizu, T. Onda, and Y. Sakamoto, "Calculation of gammaray buildup factors up to depths of $100 \mathrm{mfp}$ by the method of invariant embedding, (III) generation of an improved data set," Journal of Nuclear Science and Technology, vol. 41, no. 4, pp. 413424, 2004.

[13] Y. Harima, Y. Sakamoto, S. Tanaka, and M. Kawai, "Validity of the geometric-progression formula in approximating gammaray buildup factors," Nuclear Science and Engineering, vol. 94, no. 1, pp. 24-35, 1986.

[14] S. Singh, S. S. Ghumman, C. Singh, K. S. Thind, and G. S. Mudahar, "Buildup of gamma ray photons in flyash concretes: a study," Annals of Nuclear Energy, vol. 37, no. 5, pp. 681-684, 2010.

[15] G. S. Brar, K. Singh, M. Singh, and G. S. Mudahar, "Energy absorption buildup factor studies in water, air and concrete up to $100 \mathrm{mfp}$ using G-P fitting formula," Radiation Physics and Chemistry, vol. 43, no. 6, pp. 623-627, 1994.

[16] V. P. Singh and N. M. Badiger, "Comprehensive study of energy absorption and exposure buildup factor for concrete shielding in photon energy range $0.015-15 \mathrm{MeV}$ upto $40 \mathrm{mfp}$ penetration depth: dependency of density, chemical element, photon energy," International Journal of Nuclear Energy Science and Technology, vol. 7, no. 1, pp. 75-99, 2012.

[17] V. P. Singh and N. M. Badiger, "Photon energy absorption buildup factor of gaseous mixtures used in radiation detectors," Radioprotection, vol. 48, no. 1, pp. 63-78, 2013.

[18] M. Kurudirek, B. Doĝan, M. Ingeç, N. Ekinci, and Y. Özdemir, "Gamma-ray energy absorption and exposure buildup factor studies in some human tissues with endometriosis," Applied Radiation and Isotopes, vol. 69, no. 2, pp. 381-388, 2011.

[19] G. Sandeep and S. S. Gurdeep, "A comprehensive study on energy absorption and exposure buildup factors for some soils and ceramic materials," Journal of Applied Physics, vol. 2, no. 3, pp. 24-30, 2012.

[20] S. Tejbir, K. Gurpreet, and S. S. Parjit, "Study of gamma ray exposure buildup factor for some ceramics with photon energy, penetration depth and chemical composition," Journal of Ceramics, vol. 2013, Article ID 721606, 6 pages, 2013.

[21] E. Yilmaz, H. Baltas, E. Kiris, I. Ustabas, U. Cevik, and A. M. ElKhayatt, "Gamma ray and neutron shielding properties of some concrete materials," Annals of Nuclear Energy, vol. 38, no. 10, pp. 2204-2212, 2011.

[22] Building Materials in India: 50 Years: A Commemorative Volume, 1998. 
[23] Eco-Friendly building materials and technologies, Ecohousing assessment criteria-Version II, Annexure-4, August 2009.

[24] P. David and B. Dalibor, "Barite mortar with fluid fly ash as shielding material," Intersections, vol. 6, no. 2, article 3, pp. 28 34, 2009.

[25] S. Singh, A. Kumar, D. Singh, K. S. Thind, and G. S. Mudahar, "Barium-borate-flyash glasses: as radiation shielding materials," Nuclear Instruments and Methods in Physics Research B, vol. 266, no. 1, pp. 140-146, 2008.

[26] T. Suparat, K. Jakrapong, L. Pichet, and C. Weerapong, "Development of $\mathrm{BaO}: \mathrm{B}_{2} \mathrm{O}_{3}$ : flyash glass system for gamma-rays shielding materials," Progress in Nuclear Science and Technology, vol. 1, pp. 110-113, 2011.

[27] S. S. Amritphale, A. Anshul, N. Chandra, and N. Ramakrishnan, "Development of celsian ceramics from fly ash useful for Xray radiation-shielding application," Journal of the European Ceramic Society, vol. 27, no. 16, pp. 4639-4647, 2007.

[28] A. P. Singh, A. S. Kumar, A. Chandra, and S. K. Dhawan, "Conduction mechanism in Polyaniline-flyash composite material for shielding against electromagnetic radiation in X-band $\mathrm{Ku}$ band," AIP Advances, vol. 1, no. 2, Article ID 022147, 2011.

[29] L. Gerward, N. Guilbert, K. B. Jensen, and H. Levring, "X-ray absorption in matter. Reengineering XCOM," Radiation Physics and Chemistry, vol. 60, no. 1-2, pp. 23-24, 2001.

[30] L. Gerward, N. Guilbert, K. B. Jensen, and H. Levring, "WinXCom-a program for calculating X-ray attenuation coefficients," Radiation Physics and Chemistry, vol. 71, no. 3-4, pp. 653-654, 2004.

[31] Y. Harima, "An approximation of gamma-ray buildup factors by modified geometrical progression," Nuclear Science and Engineering, vol. 83, no. 2, pp. 299-309, 1983.

[32] M. J. Maron, Numerical Analysis: A Practical Approach, Macmillan, New York, NY, USA, 2007.

[33] G. Samuel and S. Alexander, Nuclear Reactor Engineering, vol. 1, Chapman and Hall, 4th edition, 2004.

[34] M. F. Kaplan, Concrete Radiation Shielding, Longman Scientific and Technology, Essex, UK, 1989.

[35] A. B. Chilten, J. K. Shultis, and R. E. Faw, Principle of Radiation Shielding, Prentice-Hall, Englewood Cliffs, NJ, USA, 1984.

[36] D. Luis, Update to ANSI/ANS-6.4.3-1991 for Low-Z Materials and Compound Materials and Review of Particle Transport Theory, UNLV, Las Vegas, Nev, USA, 2009. 

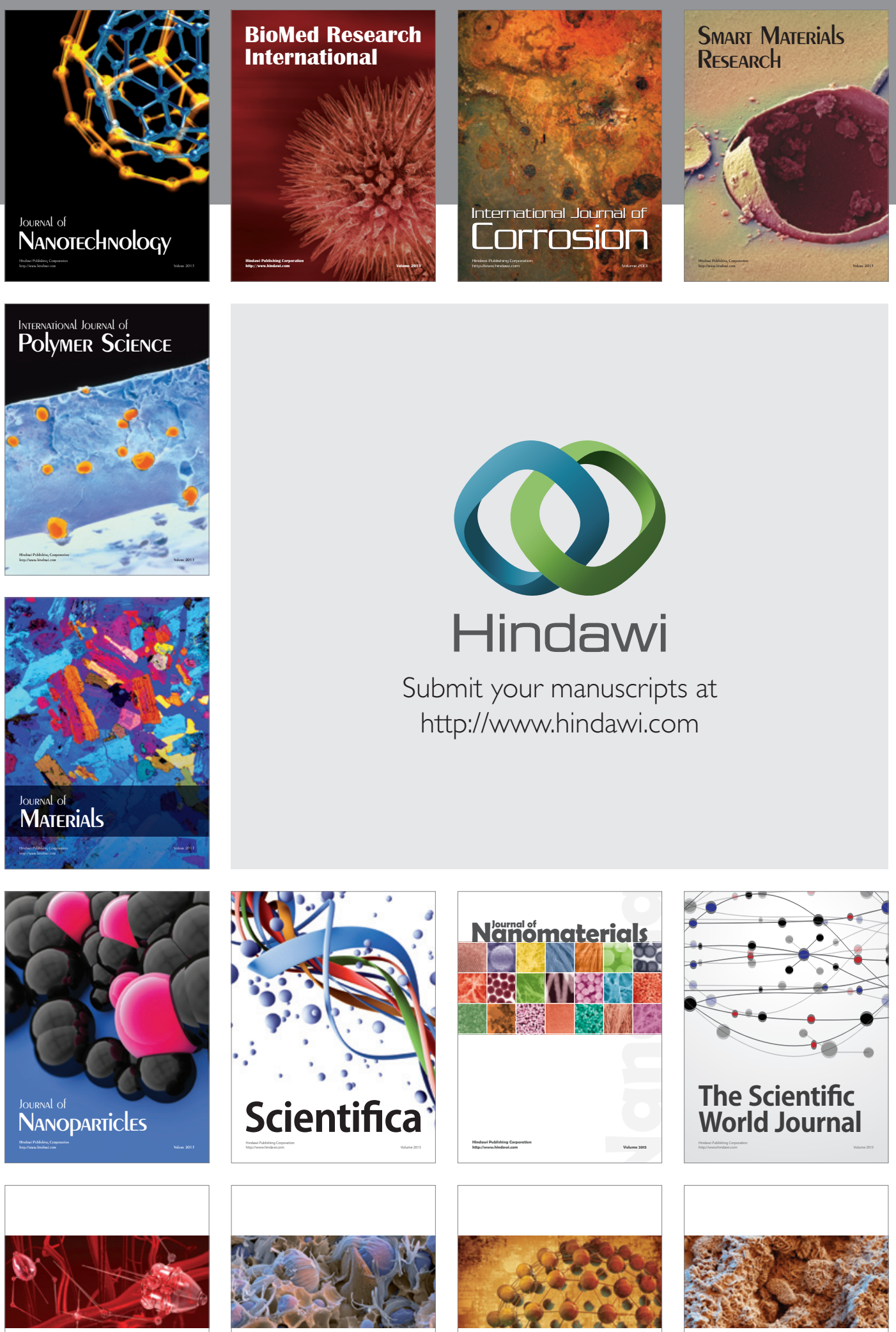

ISRN

Nanotechnology
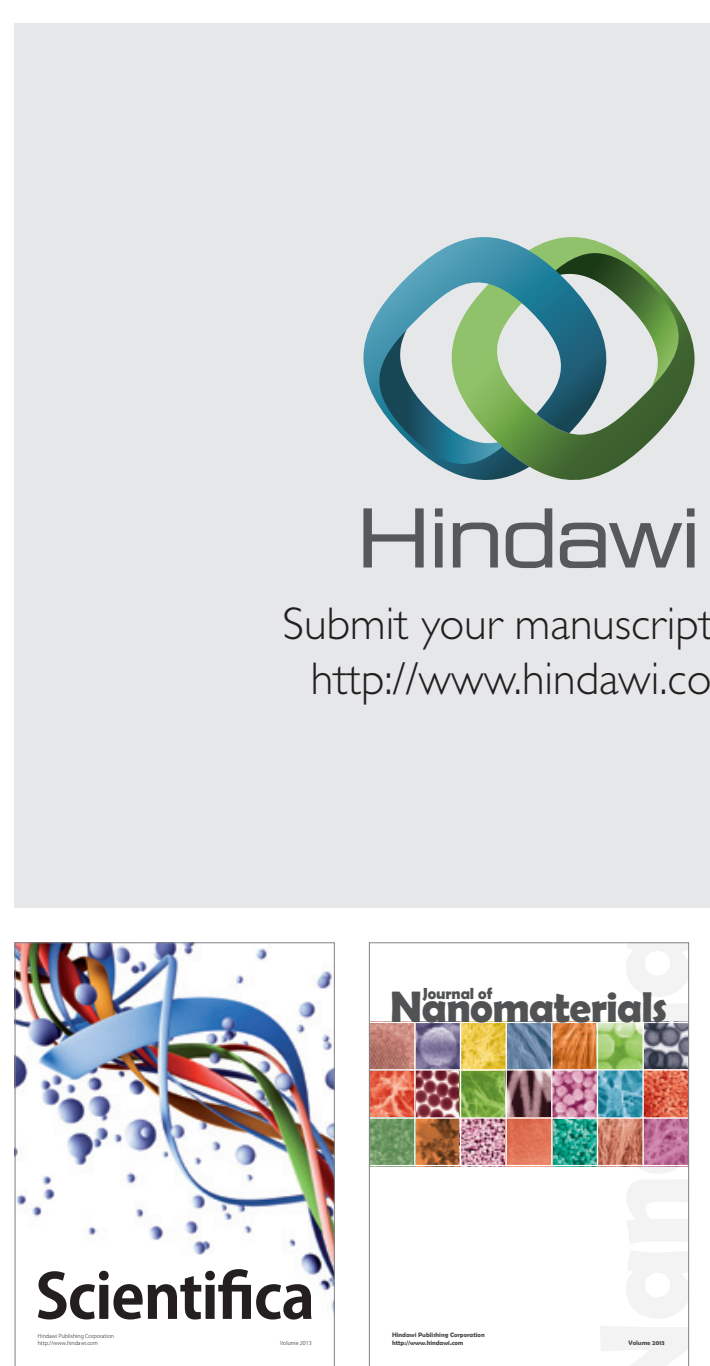

Submit your manuscripts at http://www.hindawi.com
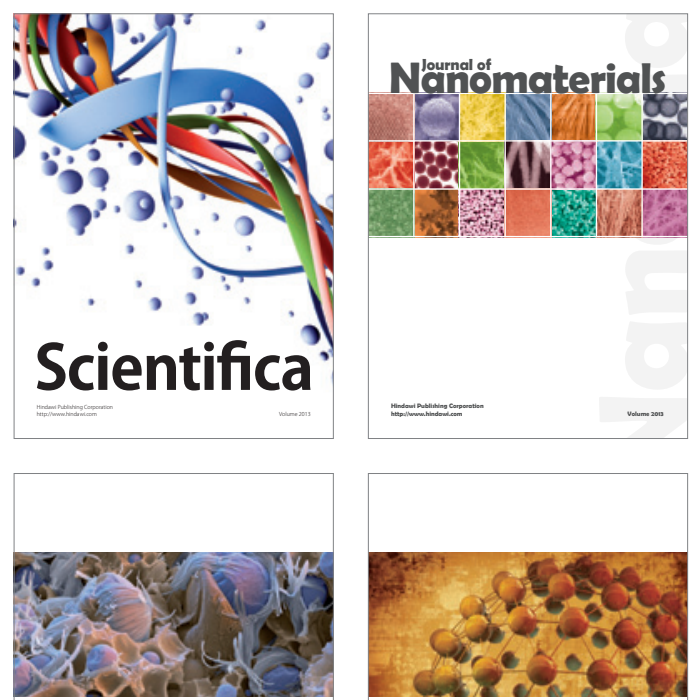

ISRN

Polymer Science

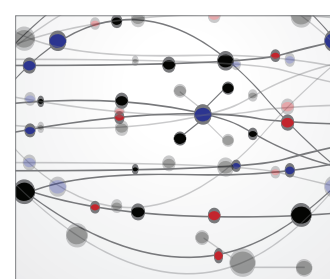

The Scientific World Journal

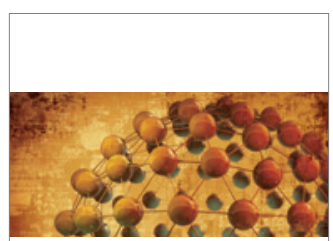

ISRN

Materials Science

\section{World Jounal}

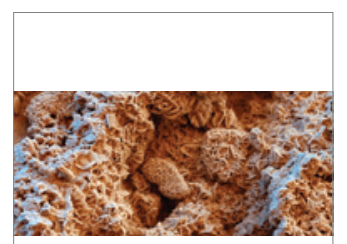

ISRN

Corrosion
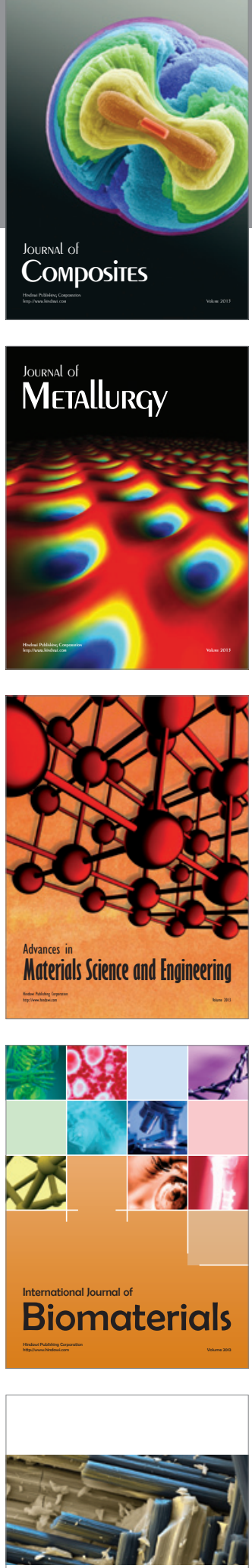

ISRN

Ceramics 